\title{
BGG CATEGORIES IN PRIME CHARACTERISTICS
}

\author{
HENNING HAAHR ANDERSEN
}

\begin{abstract}
Let $\mathfrak{g}$ be a simple complex Lie algebra. In this paper we study the BGG category $\mathcal{O}_{q}$ for the quantum group $U_{q}(\mathfrak{g})$ with $q$ being a root of unity in a field $K$ of characteristic $p>0$. We first consider the simple modules in $\mathcal{O}_{q}$ and prove a Steinberg tensor product theorem for them. This result reduces the problem of determining the corresponding irreducible characters to the same problem for a finite subset of finite dimensional simple modules. Then we investigate more closely the Verma modules in $\mathcal{O}_{q}$. Except for the special Verma module, which has highest weight $-\rho$, they all have infinite length. Nevertheless, we show that each Verma module has a certain finite filtration with an associated strong linkage principle. The special Verma module turns out to be both simple and projective/injective. This leads to a family of projective modules in $\mathcal{O}_{q}$, which are also tilting modules. We prove a reciprocity law, which gives a precise relation between the corresponding family of characters for indecomposable tilting modules and the family of characters of simple modules with antidominant highest weights. All these results are of particular interest when $q=1$, and we have paid special attention to this case.
\end{abstract}

\section{INTRODUCTION}

The BGG-category $\mathcal{O}$ for a semisimple complex Lie algebra $\mathfrak{g}$ has been studied intensively (see e.g. [21] and the large number of references there) ever since it was introduced by Bernstein, Gelfand and Gelfand, [10]. Also the corresponding version for quantum groups over $\mathbb{C}$ has been the subject of several papers, see e.g. [7], [16], [18], and [20].

On the contrary rather little can be found in the literature about BGG-categories over fields of characteristic $p>0$ (the only publication in this direction which comes to mind is [19] 1 ). Our focus in this paper is on this case - both the modular category $\overline{\mathcal{O}}_{p}$ and the quantum case $\mathcal{O}_{q}$, where the quantum parameter is a root of unity of odd order $\ell$ in a characteristic $p$ field.

We prove some basic results for both these categories. Our first goal is to understand the simple modules. We prove (Section 3) a general form of Steinberg's tensor product theorem, which was first established by Steinberg, [32, in the framework of finite dimensional modular representations of a semisimple algebraic group. Then we demonstrate, how one from this result can extract the characters of all simple modules in $\overline{\mathcal{O}}_{p}$ and $\mathcal{O}_{q}$, once the characters of all the finite dimensional simple modules are available. This last information is now determined for $\overline{\mathcal{O}}_{p}$ in terms of the socalled $p$-Kazhdan-Lusztig polynomials by the recent breakthrough in modular representation theory, [1, [29] (combined with [31] when $p<2(h-1)$ ), whereas for

\footnotetext{
${ }^{1}$ S. Donkin has kindly pointed out to me in a mail of March 28, 2022 that some of the results in this paper (in particular the facts that $\Delta_{q}(-\rho)$ is irreducible and projective in $\mathcal{O}_{q}$ (Section 6), the results about the socles of Verma modules in Section 8.1, the existence of certain projective modules in $\mathcal{O}_{q}$, Section 8.2, and the reciprocity laws in Section 8.3) were obtained several years ago by his Ph.D-student Jonathan Dixon, see his Thesis from University of London, June 2008
} 
$\mathcal{O}_{q}$ the same is so far only known to be the case for $p \gg 0$ (where the answer is given via the usual Kazhdan-Lusztig polynomials, cf. Proposition 3.14 below).

We then examine the Verma modules. Except for the special one - the Verma module with highest weight $-\rho$ - they all have infinite length unlike the situation in the ordinary category $\mathcal{O}$ and the characteristic 0 quantum case (see [7]). However, they all have finite $\left(p^{r}, \Delta\right)$-filtrations, respectively $(\ell, \Delta)$-filtrations, see Section 4 , and we show that the highest weights of the quotients in these filtrations are strongly linked to the highest weight of the Verma module in question.

We show that the special Verma module is simple (in fact the only simple Verma module), and we prove that it is also projective (Section 6). This last fact leads us to the construction of a family of indecomposable projective modules. They are projective covers of the simple modules with antidominant highest weights. We point out that this makes the simple modules in $\overline{\mathcal{O}}_{p}$ and $\mathcal{O}_{q}$ with antidominant highest weights play a special role.

The categories $\overline{\mathcal{O}}_{p}$ and $\mathcal{O}_{q}$ contain two kinds of tilting modules. First there are the finite dimensional tilting modules which have filtrations by Weyl and dual Weyl modules, see [14] and [2. Then there are also tilting modules of infinite dimension, namely those allowing (finite!) filtrations by Verma and dual Verma modules (Section 7). We call these $\infty$-tilting modules, and show that for each weight $\lambda$ in the closure of the dominant chamber, there is a unique indecomposable such module with highest weight $\lambda$. We obtain (for $p \geq 2 h-2$ ) a precise relation between the $\infty$-tilting modules and certain finite dimensional tilting modules with highest weights far inside the dominant chamber.

We also prove (Section 8 ) that in fact the indecomposable $\infty$-tilting modules coincide with the projective covers of the antidominant simple modules. This leads to a reciprocity law between the number of occurrences of a Verma module in a given indecomposable $\infty$-tilting module with dominant highest weight and the multiplicity of the corresponding antidominant simple module in the $\left(p^{r}, \Delta\right)$-filtration, respectively the $(\ell, \Delta)$-filtration of the Verma module.

Some 10 years ago when working with V. Mazorchuk on the characteristic 0 quantum BGGcategory resulting in the paper [9], I promised myself one day to investigate the characteristic $p$ analogue of such categories. When P. Fiebig's recent preprint [17] resulted in some email correspondence with him, I was reminded of this promise, and this got me started on the present work. I thank Peter for sending me his work and thus re-stimulating my interest. I am also grateful to the referee for his/her helpful comments.

\section{BGG-CATEGORIES FOR QUANTUM GROUPS AT ROOTS OF 1}

Let $K$ be a field of characteristic $p \geq 0$ and fix $q \in K^{\times}$. In this section we begin by recalling the definition of the quantum group $U_{q}$ over $K$ obtained via Lusztig's quantum divided power construction. Then we define the (integral) $B G G$-category $\mathcal{O}_{q}$ for $U_{q}$ and introduce the Verma modules in $\mathcal{O}_{q}$. This leads to the standard classification of simple modules in $\mathcal{O}_{q}$.

2.1. The quantum group $U_{q}$ over $K$. Let $\mathfrak{g}$ be a simple complex Lie algebra with Cartan matrix $C$ and denote by $v$ an indeterminate. The "generic" quantum group $U_{v}=U_{v}(\mathfrak{g})$ associated to $\mathfrak{g}$ is the $\mathbb{Q}(v)$-algebra with generators $E_{i}, F_{i}, K_{i}, K_{i}^{-1}, i=1,2, \cdots, n=\operatorname{rank} \mathfrak{g}$ and relations as given e.g. in [22], Chapter 5. This algebra has also a Hopf algebra structure, which will carry over to the $K$-algebra $U_{q}$ (see [8], Section 0) that we now define. 
Set $A=\mathbb{Z}\left[v, v^{-1}\right]$. Let $d \in \mathbb{Z}$ be non-zero. Then we have quantum numbers $[r]_{d}=$ $\frac{v^{d r}-v^{-d r}}{v^{d}-v^{-d}} \in A$ for all $r \in \mathbb{Z}$. If $r>1$ we set $[r]_{d} !=[r]_{d}[r-1]_{d} \cdots[1]_{d}$.

Choose now a diagonal matrix $D$ with diagonal entries $d_{1}, d_{2},, \cdots, d_{n} \in \mathbb{Z}_{>0}$, which are minimal with the property that $D C$ is symmetric. Then we set

$$
E_{i}^{(r)}=\frac{E_{i}^{r}}{[r]_{d_{i}} !} \text { and } F_{i}^{(r)}=\frac{F_{i}^{r}}{[r]_{d_{i}} !} \text {. }
$$

We now define $U_{A}$ to be the $A$-subalgebra of $U_{v}$ generated by $E_{i}^{(r)}, F_{i}^{(r)}, K_{i}, K_{i}^{-1}, \quad i=$ $1,2,, \cdots, n, r \in \mathbb{Z}_{>0}$.

When $q \in K^{\times}$we make $K$ into an $A$-algebra via the homomorphism $A \rightarrow K$ which takes $v$ to $q$. We define

$$
U_{q}=U_{A} \otimes_{A} K
$$

This is the quantum group (Hopf algebra) over $K$, which we shall work with. We will often abuse notation and write $E_{i}^{(r)}$ instead of $E_{i}^{(r)} \otimes 1 \in U_{q}$ etc.

We have the triangular decomposition

$$
U_{q}=U_{q}^{-} U_{q}^{0} U_{q}^{+}
$$

with $U_{q}^{-}$, respectively $U_{q}^{+}$, respectively $U_{q}^{0}$ being the subalgebra generated by all $F_{i}^{(r)}$, respectively. $E_{i}^{(r)}$, respectively $K_{i}, K_{i}^{-1},\left[\begin{array}{c}K_{i} \\ m\end{array}\right]$. Here $\left[\begin{array}{c}K_{i} \\ m\end{array}\right]$ is the specialization at $q$ of the element $\prod_{s=1}^{m} \frac{K_{i} v^{d_{i}(1-s)}-K_{i}^{-1} v^{d_{i}(s-1)}}{v^{d_{i} s}-v^{-d_{i} s}} \in U_{A}^{0}$.

We set $B_{q}=U_{q}^{0} U_{q}^{+}$.

2.2. The BGG-categories $\mathcal{O}_{q}$. Set $X=\mathbb{Z}^{n}$ and let $R \subset X \otimes_{\mathbb{Z}} \mathbb{R}$ be the root system associated with $C$. We choose a set of simple roots $S=\left\{\alpha_{1}, \alpha_{2}, \cdots, \alpha_{n}\right\}$ in $R$ and denote by $R^{+}$the corresponding set of positive roots. The ordering on $X$ induced by $R^{+}$is denoted $\leq$. By $N$ we denote the number of positive roots. The generators $E_{i}$ and $F_{i}$ above correspond to $\alpha_{i}$, resp. $-\alpha_{i}$.

We call the elements of $X$ weights and define as usual the set of dominant weights

$$
X^{+}=\left\{\lambda \in X \mid\left\langle\lambda, \alpha_{i}^{\vee}\right\rangle \in \mathbb{Z}_{\geq 0} \text { for all } i=1,2, \cdots, n\right\} .
$$

Here $\beta^{\vee}$ is the dual root of $\beta \in R$. We let $R^{\vee}$ denote the dual root system.

Let $\lambda \in X$ and set $\lambda_{i}=\left\langle\lambda, \alpha_{i}^{\vee}\right\rangle$. Then $\lambda$ defines a character

$$
\chi_{\lambda}: U_{q}^{0} \rightarrow K
$$

which takes $K_{i}$ to $q^{d_{i} \lambda_{i}}$ and $\left[\begin{array}{c}K_{i} \\ m\end{array}\right]$ to $\left(\begin{array}{c}\lambda_{i} \\ m\end{array}\right)$, [8], Lemma 1.1. We extend $\chi_{\lambda}$ to $B_{q}$ by setting $\chi_{\lambda}\left(E_{i}^{(r)}\right)=0$ for all $i=1,2, \cdots, n, r \in \mathbb{Z}_{>0}$.

For any $U_{q}^{0}$-module $M$ we define the $\lambda$-weight space in $M$ to be

$$
M_{\lambda}=\left\{m \in M \mid u m=\chi_{\lambda}(u) m, u \in U_{q}^{0}\right\} .
$$

We say that $M$ is a weight module if

$$
M=\bigoplus_{\lambda \in X} M_{\lambda}
$$


and we call $\lambda$ a weight of $M$ if $M_{\lambda} \neq 0$. Now we are ready to define the $B G G$-category we are going to study.

Definition 2.1. The category $\mathcal{O}_{q}$ is the full subcategory of the category of $U_{q}$-modules consisting of those $M$, which satisfy

(1) $M$ is a weight module,

(2) $\operatorname{dim}_{k}\left(M_{\lambda}\right)<\infty$ for all $\lambda$, and there exist finitely many $\lambda_{1}, \lambda_{2}, \cdots, \lambda_{r} \in X$ such that for any weight $\lambda$ of $M$ we have $\lambda \leq \lambda_{i}$ for some $i \in\{1,2, \cdots, r\}$,

(3) for all $m \in M$ we have $\operatorname{dim}_{K}\left(B_{q} m\right)<\infty$.

Remark 2.2. In [21] (as well as in [7]) the BGG-category is defined to consist of finitely generated $U_{K}$ (or $U_{q}$ ) -modules. We have chosen the weaker finiteness condition (2), because it ensures that $\mathcal{O}_{q}$ is an abelian category for all $p$. Moreover, it also implies that the duality functor (as defined as in [21], Section 3.3) preserves $\mathcal{O}_{q}$.

Note that $\mathcal{O}_{q}$ consists of modules with integral weights. Moreover, our definition of weight modules implies that all modules in $\mathcal{O}_{q}$ have type $\mathbf{1}$.

When $M \in \mathcal{O}_{q}$, the character $\operatorname{ch} M$ is given by

$$
\operatorname{ch} M=\sum_{\lambda \in X} \operatorname{dim}_{K}\left(M_{\lambda}\right) e^{\lambda} .
$$

2.3. Verma modules and simple modules in $\mathcal{O}_{q}$. Let $\lambda \in X$. Exactly as in the classical case we define the Verma module $\Delta_{q}(\lambda) \in \mathcal{O}_{q}$ by

$$
\Delta_{q}(\lambda)=U_{q} \otimes_{B_{q}} \lambda
$$

where $\lambda$ denotes the 1 -dimensional $K$-space with $B_{q}$-action given by $\chi_{\lambda}$.

Note that $\Delta_{q}(\lambda)_{\mu} \neq 0$ iff $\mu \leq \lambda$. Moreover, $\operatorname{dim}_{K} \Delta_{q}(\lambda)_{\lambda}=1$.

As $U_{q}$ has a $P B W$-bases we see that the character of $\Delta_{q}(\lambda)$ is given by

$$
\operatorname{ch} \Delta_{q}(\lambda)=\sum_{\mu \leq \lambda} P(\lambda-\mu) e^{\mu} .
$$

Here $P$ is the Kostant partition function.

We set $q^{-}=\operatorname{ch} \Delta_{q}(-\rho)=\sum_{\mu \leq-\rho} P(-\rho-\mu) e^{\mu}$. Then by the above formula we get for arbitrary $\lambda \in X$

$$
\operatorname{ch} \Delta_{q}(\lambda)=q^{-} e^{\lambda+\rho}
$$

Remark 2.3. Here we are using a notation similar to the one used in [21]. In fact, our $q^{-}$ is the inverse of Humphreys $q$-function (see [21], Section 2.3). Note that the notation $q^{-}$has nothing to do with the quantum parameter $q$.

Clearly $\Delta_{q}(\lambda)$ has a unique maximal proper submodule, namely the sum of all submodules $M \subset \Delta_{q}(\lambda)$ with $M_{\lambda}=0$. In other words, $\Delta_{q}(\lambda)$ has a unique simple quotient. We shall denote this quotient $L_{q}(\lambda)$.

Theorem 2.4. (1) The set $\left\{L_{q}(\lambda) \mid \lambda \in X\right\}$ is up to isomorphisms a complete list of simple modules in $\mathcal{O}_{q}$.

(2) $L_{q}(\lambda)$ is finite dimensional iff $\lambda \in X^{+}$. 
(3) Let $\lambda \in X^{+}$. If $q^{m} \neq 1$ for all $m>0$ then $\operatorname{ch} L_{q}(\lambda)=\chi(\lambda)$, where $\chi(\lambda)$ is the Weyl character.

Proof. (1) and (2) are proved exactly as in the classical case, [21, Sections 1.3 and 1.6. The proof of (3) is given in [8], Theorem 6.4.

Remark 2.5. When $p=0$ the equality in (3) also holds for $q= \pm 1$, see [8], Remark following Theorem 6.4.

\section{Steinberg's tensor Product theorem in $\mathcal{O}_{q}$}

We continue to denote by $K$ an arbitrary field. In this section $q \in K$ will be a root of unity of order $\ell$. For convenience we shall assume $\ell$ is odd (to extend to even $\ell$ see [3] and [6]) and not divisible by 3 when $R$ is of type $G_{2}$. When $q=1$ and $p=0$ the category $\mathcal{O}_{q}$ is the ordinary category $\mathcal{O}$. The case $\ell>1$ and $p=0$ was treated in [9]. So in this paper we focus on $p>0$. When $\ell=1$ we may identify $\mathcal{O}_{q}$ with the modular category $\overline{\mathcal{O}}_{p}$ for the hyperalgebra of $\mathfrak{g}$. In the following we will always assume $\ell>1$ (i.e. $q$ a non-trivial root of 1 ), using special notation (bars and subscript $p$ ) when we are in the modular category, i.e. when $q=1$.

3.1. The small quantum group. The small quantum group $u_{q}$ is the subalgebra of $U_{q}$ generated by $E_{i}, F_{i}, K_{i}, i=1,2,, \cdots, n$, cf. [28]. Just like for $U_{q}$ we have a triangular decomposition $u_{q}=u_{q}^{-} u_{q}^{0} u_{q}^{+}$. As $E_{i}^{\ell}=[\ell]_{d_{i}} ! E_{i}^{(\ell)}=0$ (because by our assumption $[\ell]_{d_{i}}=0$ ) we see that $u_{q}^{+}$is finite dimensional. In fact, via the PBW-bases we obtain $\operatorname{dim} u_{q}^{+}=\ell^{N}$, where $N$ is the number of positive roots, cf. the analogous modular case treated in [23], II.3.3. Likewise, $\operatorname{dim} u_{q}^{-}=\ell^{N}$. As $K_{i}^{2 \ell}=1$ we have also that $u_{q}^{0}$ (and hence $u_{q}$ ) is finite dimensional.

It will be convenient for us instead of $u_{q}$-modules to consider finite dimensional modules for the subalgebra $u_{q} B_{q}$ of $U_{q}$. Note that $u_{q} B_{q}$ has the triangular decomposition $u_{q} B_{q} \simeq$ $u_{q}^{-} \otimes U_{q}^{0} \otimes U_{q}^{+}$.

3.2. Baby Verma modules and simple $u_{q} B_{q}$-modules. Let $\lambda \in X$. Like in Section 2 we consider $\lambda$ as a 1-dimensional $B_{q}$-module.

The baby Verma module corresponding to $\lambda$ is defined by

$$
\tilde{\Delta}_{\ell}(\lambda)=u_{q} B_{q} \otimes_{B_{q}} \lambda .
$$

By the facts recalled in Section 3.1 we have $\operatorname{dim} \tilde{\Delta}_{\ell}(\lambda)=\ell^{N}$. Moreover, the fact that $u_{q} B_{q}=$ $u_{q}^{-} \otimes B_{q}$ implies that weight multiplicities in baby Verma modules are given by

$$
\operatorname{dim}_{K} \tilde{\Delta}_{\ell}(\lambda)_{\lambda-\mu}=\#\left\{\left(n_{\beta}\right)_{\beta \in R^{+}} \mid 0 \leq n_{\beta}<\ell \text { for all } \beta \text { and } \sum_{\beta \in R^{+}} n_{\beta} \beta=\mu\right\}
$$

This formula is identical to its modular analogue in [23], Section II.9.2.

Note that for any $\mu \in X$ the character $\ell \mu$ of $B_{q}$ extends to a character of $u_{q} B_{q}$. In fact, $\ell \mu$ restricts to the trivial character on $u_{q}^{0}$. So $\ell \mu$ extends from $B_{q}$ to $u_{q} B_{q}$ by defining it to be trivial on $u_{q}$.

The same arguments as in Section 2.3 show that $\tilde{\Delta}_{\ell}(\lambda)$ has a unique simple quotient, and if we denote this $\tilde{L}_{\ell}(\lambda)$, then the set of simple modules $\left(\tilde{L}_{\ell}(\lambda)\right)_{\lambda \in X}$ is up to isomorphism a full set of non-isomorphic finite dimensional $u_{q} B_{q}$-modules (of type $\mathbf{1}$ ). 
By the tensor identity (or just from the definition of $\tilde{\Delta}_{\ell}$ ) combined with the above observation about $\ell$-multiples of characters in $X$ we get

$$
\tilde{\Delta}_{\ell}(\lambda+\ell \mu) \simeq \tilde{\Delta}_{\ell}(\lambda) \otimes \ell \mu \text { and } \tilde{L}_{\ell}(\lambda+\ell \mu)=\tilde{L}_{\ell}(\lambda) \otimes \ell \mu \text { for all } \lambda, \mu \in X .
$$

Set now

$$
X_{\ell}=\left\{\lambda \in X^{+} \mid\left\langle\lambda, \alpha_{i}^{\vee}\right\rangle<\ell \text { for all } i=1,2, \cdots, n\right\} .
$$

This is the set of $\ell$-restricted weights. An important result (the quantum analogue of Curtis' theorem, [13], for the algebraic group over $K$ corresponding to $\mathfrak{g}$ ) says that the simple $u_{q^{-}}$ modules are in fact restrictions of the simple $U_{q}$-modules in $\mathcal{O}_{q}$ with $\ell$-restricted highest weights (see [9], Theorem 1.9).

Theorem 3.1. If $\lambda \in X_{\ell}$ then $L_{q}(\lambda)$ remains simple when restricted to $u_{q}$, i.e. we have $L_{q}(\lambda)_{\left.\right|_{u_{q}}} \simeq L_{\ell}(\lambda)$.

An immediate consequence of this theorem and the second part of (3.2) is

Corollary 3.2. Let $\lambda \in X$ and write $\lambda=\lambda^{0}+\ell \lambda^{1}$ for (unique) $\lambda^{0} \in X_{\ell}$ and $\lambda^{1} \in X$. Then

$$
\tilde{L}_{\ell}(\lambda) \simeq L_{q}\left(\lambda^{0}\right)_{\left.\right|_{u_{q} B_{q}}} \otimes \ell \lambda^{1} .
$$

3.3. The Steinberg module for $U_{q}$. The Steinberg module for $U_{q}$ is

$$
S t_{q}=L_{q}((\ell-1) \rho),
$$

where $\rho$ as usual denotes half the sum of the positive roots in $R$. By the strong linkage principle for quantum groups at roots of 1, [6], Corollary 4.5, we have also $S t_{q}=H_{q}^{0}((\ell-1) \rho)$. Here $H_{q}^{0}((\ell-1) \rho)$ is the dual Weyl module with highest weight $(\ell-1) \rho$. Its character is $\chi((\ell-1) \rho)$, and it has dimension $\ell^{N}$. We conclude that when we restrict to $u_{q} B_{q}$ we have

$$
S t_{q}=\tilde{\Delta}_{q}((\ell-1) \rho)=\tilde{L}_{q}((\ell-1) \rho) .
$$

Later we shall need the following result.

Proposition 3.3. Let $\lambda \in X_{\ell}$. Then

$$
L_{\ell}(\lambda)=\Delta_{\ell}(\lambda) \text { iff } \lambda=(l-1) \rho .
$$

Proof. By Theorem 3.1 we have $L_{\ell}(\lambda)=L_{q}(\lambda)_{\left.\right|_{q}}$. Now $L_{q}(\lambda)$ is a submodule of the dual Weyl module $H_{q}^{0}(\lambda)$. Therefore, $\operatorname{dim}_{K} L_{\ell}(\lambda) \leq \operatorname{dim}_{K} H_{q}^{0}(\lambda)$. But the latter dimension is given by Weyl's dimension formula and it is immediate that this is strictly less than $\ell^{N}$ if $\lambda \in X_{\ell} \backslash\{(\ell-1) \rho\}$.

3.4. The quantum Frobenius homomorphism. Let $\bar{U}=U_{\mathbb{Q}}(\mathfrak{g})$ be the ordinary enveloping algebra of $\mathfrak{g}$ over $\mathbb{Q}$. We choose the standard Chevalley generators $e_{i}, f_{i}, h_{i}, i=1,2, \cdots, n$ for $\mathfrak{g}$. Via the corresponding Kostant $\mathbb{Z}$-form $\bar{U}_{\mathbb{Z}}$ in $\bar{U}$ we obtain the hyperalgebra $\bar{U}_{K}=$ $\bar{U}_{\mathbb{Z}} \otimes_{\mathbb{Z}} K$ for $\mathfrak{g}$ over $K$. There is a quantum Frobenius homomorphism (see [28], Section 8, 9], $1.2)$

$$
F_{q}: U_{q} \rightarrow \bar{U}_{K}
$$


which is determined by

and

$$
\begin{gathered}
F_{q}\left(E_{i}^{(r)}\right)=\left\{\begin{array}{l}
e_{i}^{\left(\frac{r}{\ell}\right)} \text { if } \ell \mid r, \\
0 \text { otherwise },
\end{array}\right. \\
F_{q}\left(F_{i}^{(r)}\right)=\left\{\begin{array}{l}
f_{i}^{\left(\frac{r}{\ell}\right)} \text { if } \ell \mid r, \\
0 \text { otherwise },
\end{array}\right. \\
F_{q}\left(K_{i}\right)=1,
\end{gathered}
$$

$$
F_{q}\left(\left[\begin{array}{c}
K_{i} \\
m
\end{array}\right]\right)=\left\{\begin{array}{l}
\left(\begin{array}{c}
h_{i} \\
\frac{m}{\ell}
\end{array}\right) \text { if } \ell \mid m, \\
0 \text { otherwise. }
\end{array}\right.
$$

This allows us to make any $\bar{U}_{K}$-module $M$ into a $U_{q}$-module. We denote the $U_{q}$-module obtained in this way by $M^{[\ell]}$. Note that $u_{q}$ acts trivially on $M^{[\ell]}$. On the other hand, if $L$ is a $U_{q}$-module which is trivial when restricted to $u_{q}$, then there exists a $\bar{U}_{K}$-module $\bar{L}$ such that $L=\bar{L}^{[\ell]}$. In this case we write $\bar{L}=L^{[-\ell]}$.

3.5. The quantum Steinberg tensor product theorem in $\mathcal{O}_{q}$. We define the $B G G$ category $\overline{\mathcal{O}}_{p}$ for $\bar{U}_{K}$ just like we defined $\mathcal{O}_{q}$ for $U_{q}$ in Section 2.2. The Verma modules in $\overline{\mathcal{O}}_{p}$ are denoted $\bar{\Delta}_{p}(\lambda), \lambda \in X$, and the simple quotient of $\bar{\Delta}_{p}(\lambda)$ is $\bar{L}_{p}(\lambda)$. When $p=0$ we are in the ordinary integral category situation, i.e. we have $\overline{\mathcal{O}}_{0}=\mathcal{O}_{\text {int }}$, see [7].

The quantum Steinberg tensor product theorem is now the following result.

Theorem 3.4. Let $\lambda \in X$ and write $\lambda=\lambda^{0}+\ell \lambda^{1}$ with $\lambda^{0} \in X_{\ell}$. Then we have an isomorphism in $\mathcal{O}_{q}$

$$
L_{q}(\lambda) \simeq L_{q}\left(\lambda^{0}\right) \otimes \bar{L}_{p}\left(\lambda^{1}\right)^{[\ell]} .
$$

Proof. When $p=0$ this was proved in [27, Theorem 7.4. We gave a different proof in [7, Theorem 3.1 (also in the case $p=0$ ). This proof carries over to arbitrary $p$.

Remark 3.5. The original Steinberg tensor product theorem, 32 dealt with finite dimensional simple modules for seminsimple algebraic groups in characteristic $p>0$. The most general quantum version for finite dimensional simple modules known to the author is [9], Theorem 1.10 .

3.6. Steinberg's tensor product theorem in $\overline{\mathcal{O}}_{p}$. In this section we assume $p>0$.

Consider the "usual" Frobenius homomorphism $\bar{F}: \bar{U}_{K} \rightarrow \bar{U}_{K}$. It is given on generators by

$$
\begin{gathered}
e_{i}^{(r)} \mapsto\left\{\begin{array}{l}
e_{i}^{\left(\frac{r}{p}\right)} \text { if } p \mid r, \\
0 \text { otherwise, }
\end{array}\right. \\
f_{i}^{(r)} \mapsto\left\{\begin{array}{l}
f_{i}^{\left(\frac{r}{p}\right)} \text { if } p \mid r, \\
0 \text { otherwise, }
\end{array}\right. \\
\left(\begin{array}{l}
h_{i} \\
m
\end{array}\right) \mapsto\left\{\begin{array}{l}
\left(\begin{array}{l}
h_{i} \\
\frac{m}{p}
\end{array}\right) \text { if } p \mid m, \\
0 \text { otherwise. }
\end{array}\right.
\end{gathered}
$$

Twisting a $\bar{U}_{K}$-module $M$ by $\bar{F}$ we obtain a new $\bar{U}_{K}$-module which we denote $M^{(1)}$. We can iterate the twisting and get in this way modules $M^{(r)}, r \geq 0$. We have corresponding 
small hyperalgebras (or $r$-restricted enveloping algebras) $\bar{u}_{r}$, namely the subalgebras generated by all the $e_{i}^{(m)}$ and $f_{i}^{(m)}$ with $m<p^{r}$.

The same arguments as in Section 3.5 lead to the following result.

Theorem 3.6. Let $\lambda \in X$ and write $\lambda=\lambda^{0}+p \lambda^{1}, \lambda^{0} \in X_{p}, \lambda^{1} \in X$. Then we have an isomorphism in $\overline{\mathcal{O}}_{p}$

$$
\bar{L}_{p}(\lambda) \simeq \bar{L}_{p}\left(\lambda^{0}\right) \otimes \bar{L}_{p}\left(\lambda^{1}\right)^{(1)} .
$$

Remark 3.7. In this theorem we have abused notation and used the same notation for the $p$-adic expression for $\lambda$ as we have earlier used for the $\ell$-adic expression. We will continue to do this in the following, sometimes also replacing $p$ by some higher $p$-power. It should be clear from the context which case we work with.

Iterating Theorem $3.6 r$-times we get

Corollary 3.8. Suppose $\lambda \in X$. Write $\lambda=\lambda^{0}+p^{r} \lambda^{1}$ with $\lambda^{0} \in X_{p^{r}}$ and $\lambda^{1} \in X$. Then in $\overline{\mathcal{O}}_{p}$ we have

$$
\bar{L}_{p}(\lambda) \simeq \bar{L}_{p}\left(\lambda^{0}\right) \otimes \bar{L}_{p}\left(\lambda^{1}\right)^{(r)} .
$$

Remark 3.9. Consider $\lambda=\left(p^{r}-1\right) \rho, r>0$. In this case we use the notation $\overline{S t}_{r}=$ $L\left(\left(p^{r}-1\right) \rho\right)$. This is the $r$-th Steinberg module in $\overline{\mathcal{O}}_{p}$. Applying Theorem 3.6 several times we get $\overline{S t}_{r}=\overline{S t}_{1} \otimes \overline{S t}_{1}^{(2)} \otimes \cdots \otimes \overline{S t}_{1}^{(r-1)}$.

3.7. Applications of the Steinberg theorems. In this section we derive several consequences of Theorems 3.4 and 3.6. First we apply Corollary 3.8 to obtain

Corollary 3.10. Let $\lambda \in X$ and $r \in \mathbb{Z}_{>0}$. Write $\lambda=\lambda^{0}+p^{r} \lambda^{1}$ with $\lambda^{0} \in X_{p^{r}}, \lambda^{1} \in X$. Fix $\mu \leq \lambda$. Then we have

for all $r \gg 0$.

$$
\operatorname{dim}_{K} \bar{L}_{p}(\lambda)_{\mu}=\operatorname{dim}_{K} \bar{L}_{p}\left(\lambda^{0}\right)_{\mu-p^{r} \lambda^{1}}
$$

Proof. By Corollary 3.8 we have

$$
\operatorname{dim}_{K} \bar{L}_{p}(\lambda)_{\mu}=\sum_{\nu, \eta} \operatorname{dim}_{K} \bar{L}_{p}\left(\lambda^{0}\right)_{\nu} \operatorname{dim}_{K} \bar{L}_{p}\left(\lambda^{1}\right)_{\eta},
$$

where the sum runs over all $\nu \leq \lambda^{0}$ and $\eta \leq \lambda^{1}$ satisfying $\nu+p^{r} \eta=\mu$. We write $\nu=$ $\lambda^{0}-\sum_{\alpha \in S} m_{\alpha} \alpha, \eta=\lambda^{1}-\sum_{\alpha \in S} k_{\alpha} \alpha$ and $\mu=\lambda-\sum_{\alpha \in S} n_{\alpha} \alpha$ with $m_{\alpha}, k_{\alpha}, n_{\alpha} \in \mathbb{Z}_{\geq 0}$. The condition $\nu+p^{r} \eta=\mu$ then means

$$
m_{\alpha}+p^{r} k_{\alpha}=n_{\alpha} \text { for all } \alpha \in S .
$$

Hence if $p^{r}>n_{\alpha}$ for all $\alpha \in S$ we must have $k_{\alpha}=0$ and $m_{\alpha}=n_{\alpha}$ for all $\alpha \in S$. In other words, the equation (3.3) contains just 1 summand on the right hand side, namely the one with $\eta=\lambda^{1}$ and $\nu=\lambda^{0}-(\lambda-\mu)=\mu-p^{r} \lambda^{1}$. This gives the stated formula.

Remark 3.11. (1) Note that the proof gives an explicit bound for how large $r$ needs to be in order for the formula in this corollary to hold for a given $\mu=\lambda-\sum_{\alpha \in S} n_{\alpha} \alpha$, namely $r>n_{\alpha}$ for all $\alpha \in S$. 
(2) The same formula was obtained by P. Fiebig, see [17], Theorem 1, with a different proof.

Corollary 3.12. The characters $\left\{\operatorname{ch} L_{q}(\lambda) \mid \lambda \in X\right\}$ of the irreducible modules in $\mathcal{O}_{q}$ are determined by the two finite subsets $\left\{\operatorname{ch} L_{q}(\lambda) \mid \lambda \in X_{\ell}\right\}$ and $\left\{\operatorname{ch} \bar{L}_{p}(\lambda) \mid \lambda \in X_{p}\right\}$.

Proof. Let $\lambda \in X$ and write $\lambda=\lambda^{0}+\ell \lambda^{1}$ with $\lambda^{0} \in X_{\ell}$ and $\lambda^{1} \in X$. By Theorem 3.4 we have $\operatorname{ch} L_{q}(\lambda)=\operatorname{ch} L_{q}\left(\lambda^{0}\right)\left(\operatorname{ch} \bar{L}_{p}\left(\lambda^{1}\right)\right)^{(\ell)}$ Here we use the notation $\left(\sum_{\mu} a_{\mu} e^{\mu}\right)^{(\ell)}=\sum_{\mu} a_{\mu} e^{\ell \mu}$. Now apply Corollary 3.10 .

Remark 3.13. The characteristic 0 analogue of this corollary says that in that case the irreducible characters in $\mathcal{O}_{q}$ are given by the finite set $\left\{c h L_{q}(\lambda) \mid \lambda \in X_{\ell}\right\}$ together with the irreducible characters in ordinary (integral) category $\mathcal{O}$, cf. [7, Section 5. The latter characters are given by the Kazhdan-Lusztig polynomials for the Weyl Group $W$ of $\mathfrak{g}$, [26], whereas the former set of characters are determined by the Kazhdan-Lusztig polynomials for the affine Weyl group $W \ltimes \mathbb{Z} R^{\vee}$, see [24] and [25]. In characteristic $p$ the situation is more complicated: the modular irreducible characters $\left\{\operatorname{ch} \bar{L}_{p}(\lambda) \mid \lambda \in X_{p}\right\}$ are determined by the $p$ Kazhdan-Lusztig polynomials (at least for $p \geq 2 h-1$ ), see [1], 29]. The quantum irreducible characters $\left\{\operatorname{ch} L_{q}(\lambda) \mid \lambda \in X_{\ell}\right\}$ are (at least for $p \gg 0$ ) identical to their characteristic 0 counterparts, as we shall now prove.

Proposition 3.14. Let $\zeta \in \mathbb{C}$ be a primitive $\ell$-th root of 1 . Then for $p \gg 0$ we have

$$
\operatorname{ch} L_{q}(\lambda)=\operatorname{ch} L_{\zeta}(\lambda)
$$

for all $\lambda \in X_{\ell}$.

This result was noticed many years ago by L. Thams in his PhD-thesis (Aarhus University, 1993). As this thesis is not easily available we have reproduced the proof below.

Proof. Let $\lambda \in X^{+}$. We shall use notation from [8]. For instance, $H_{q}^{N}\left(w_{0} \cdot \lambda\right)$ will denote the Weyl module for $U_{q}$ with highest weight $\lambda \in X^{+}$, and $H_{q}^{0}(\lambda)$ is the corresponding dual Weyl module (the restrictions on $\ell$ in [8] are no longer needed as the quantum Kempf's vanishing theorem have been proved in general, see [30]).

The irreducible module $L_{q}(\lambda)$ is the image of the natural homomorphism $c_{q}: H_{q}^{N}\left(w_{0} \cdot \lambda\right) \rightarrow$ $H_{q}^{0}(\lambda)$. Both these modules have $A$-forms, which we denote $H_{A}^{N}\left(w_{0} \cdot \lambda\right)$ and $H_{A}^{0}(\lambda)$, respectively. Also $c_{q}$ lifts to a $U_{A}$-homomorphism $c_{A}: H_{A}^{N}(w \cdot \lambda) \rightarrow H_{A}^{0}(\lambda)$. This is an injection because the corresponding homomorphism over the fraction field $\mathbb{Q}(v)$ is an isomorphism, cf [8], Theorem 6.4. Setting $C_{A}(\lambda)=\operatorname{coker}\left(c_{A}\right)$ we have the short exact sequence

$$
0 \rightarrow H_{A}^{N}\left(w_{0} \cdot \lambda\right) \rightarrow H_{A}^{0}(\lambda) \rightarrow C_{A}(\lambda) \rightarrow 0 .
$$

Tensoring this sequence by $A_{\ell}=A /\left(\Phi_{\ell}\right)$ ), where $\Phi_{\ell}$ is the $\ell$-th cyclotomic polynomial in $A$ we get the exact sequence

$$
H_{A_{\ell}}^{N}\left(w_{0} \cdot \lambda\right) \rightarrow H_{A_{\ell}}^{0}(\lambda) \rightarrow C_{A}(\lambda) \otimes_{A} A_{\ell} \rightarrow 0 .
$$

Recall that we consider $K$, respectively $\mathbb{C}$ as $A$-algebras via the homomorphisms taking $v \in A$ into $q$ and $\zeta$, respectively. Note that both these structure maps factor through $A_{\ell}$. So when 
we tensor (3.4) by $K$, respectively $\mathbb{C}$ we get exact sequences

$$
H_{q}^{N}\left(w_{0} \cdot \lambda\right) \rightarrow H_{q}^{0}(\lambda) \rightarrow C_{A_{\ell}}(\lambda) \otimes_{A_{\ell}} K \rightarrow 0,
$$

respectively

$$
H_{\zeta}^{N}\left(w_{0} \cdot \lambda\right) \rightarrow H_{\zeta}^{0}(\lambda) \rightarrow C_{A_{\ell}}(\lambda) \otimes_{A_{\ell}} \mathbb{C} \rightarrow 0
$$

These two sequences show that $\operatorname{ch} L_{q}(\lambda)=\operatorname{ch} L_{\zeta}(\lambda)$ if and only if $\operatorname{dim}_{K}\left(C_{A_{\ell}}(\lambda) \otimes_{A_{\ell}} K\right)=$ $\operatorname{dim}_{\mathbb{C}}\left(C_{A_{\ell}}(\lambda) \otimes_{A_{\ell}} \mathbb{C}\right)$. This holds in turn iff $C_{A_{\ell}}(\lambda)$ has no $p$-torsion. As $C_{A_{\ell}}(\lambda)$ is finitely generated, this will certainly be true if $p$ is big enough, say $p>m(\lambda)$ where $m(\lambda) \in \mathbb{Z}_{>0}$. We conclude that the proposition holds for $p>M(\ell)=\max \left\{m(\lambda) \mid \lambda \in X_{\ell}\right\}$.

Remark 3.15. I have no estimate for the $M(\ell)$ occurring in this proof.

3.8. Frobenius homomorphisms and Hom-space identities. For later use we record here a couple of general identities for Hom-spaces related to the quantum and the modular Frobenius homomorphisms from Section 3.5, respectively Section 3.6.

Proposition 3.16. (1) Let $M$ and $N$ be two $U_{q}$-modules and $L$ be a $\bar{U}_{K}$-module. Then the quantum Frobenius homomorphism $F_{q}$ gives an isomorphism

$$
\operatorname{Hom}_{U_{q}}\left(M \otimes L^{[\ell]}, N\right) \simeq \operatorname{Hom}_{\bar{U}_{K}}\left(L, \operatorname{Hom}_{u_{q}}(M, N)^{[-\ell]}\right) .
$$

(2) Let $L, M$ and $N$ be three $\bar{U}_{K}$-modules. Then for each $r>0$ we have an isomorphism

$$
\operatorname{Hom}_{\bar{U}_{K}}\left(M \otimes L^{(r)}, N\right) \simeq \operatorname{Hom}_{\bar{U}_{K}}\left(L, \operatorname{Hom}_{\bar{u}_{r}}(M, N)^{(-r)}\right)
$$

The identities in this proposition are the Hopf-algebra versions of the corresponding ones related to a group homomorphism $f: G \rightarrow G^{\prime}$. If $H$ denotes the kernel of $f$ then for any $G$-module $M$ we have an obvious identification $M^{G}=\left(M^{H}\right)^{G / H}$. On Hom-spaces this leads to identities

$$
\operatorname{Hom}_{G}(M \otimes L, N)=\operatorname{Hom}_{G / H}\left(L, \operatorname{Hom}_{H}(M, N)\right)
$$

for any two $G$-modules $M$ and $N$, and any $G / H$-module $L$.

\section{LINKAGE PRINCIPLES}

In this section we shall derive linkage principles for Verma modules, first in $\overline{\mathcal{O}}_{p}$ and then in $\mathcal{O}_{q}$. To state them we shall need the affine Weyl groups $W_{p}$ and $W_{\ell}$. These are the groups generated by the affine reflections $s_{\beta, m}$, where $\beta \in R^{+}$and $m \in \mathbb{Z}$, defined by

$$
s_{\beta, n} \cdot \lambda=s_{\beta} \cdot \lambda+m p \beta, \lambda \in X
$$

in the case of $W_{p}$. For $W_{\ell}$ we replace $p$ by $\ell$ in this formula. 
4.1. Linkage principles for Verma modules in $\overline{\mathcal{O}_{p}}$. We assume $p>0$ and use the notation from Section 3.

The hyperalgebra $\bar{U}_{K}$ has triangular decomposition $\bar{U}_{K}=\bar{U}_{K}^{-} \bar{U}_{K}^{0} \bar{U}_{K}^{+}$, where $\bar{U}_{K}^{-}$, respectively $\bar{U}_{K}^{+}$, is the subalgebra generated by all $f_{i}^{(r)}$, respectively $e_{i}^{(r)}$, and $\bar{U}_{K}^{0}$ is generated by all $\left(\begin{array}{c}h_{i} \\ m\end{array}\right)$. We set $\bar{B}_{K}=\bar{U}_{K}^{0} \bar{U}_{K}^{+}$. Then the Verma module in $\overline{\mathcal{O}}_{p}$ with highest weight $\lambda \in X$ is

$$
\bar{\Delta}_{p}(\lambda)=\bar{U}_{K} \otimes_{\bar{B}_{K}} \lambda \text {. }
$$

Recall from Section 3.6 that we have the small hyperalgebra $\bar{u}_{1}$ is of $\bar{U}_{K}$ generated by $f_{i}, h_{i}, e_{i} i=1,2,3, \cdots, n$., and corresponding higher versions $u_{r}$ for all $r>1$. We have then baby Verma modules defined by

$$
\tilde{\Delta}_{r}(\lambda)=\bar{u}_{r} \bar{B}_{K} \otimes_{\bar{B}_{K}} \lambda, \lambda \in X .
$$

These modules have simple quotients denoted $\tilde{L}_{p^{r}}(\lambda)$.

Recall from [23], II. 9.11 (or see [15] for a slightly stronger version) that we have the following linkage principle for baby Verma modules.

Theorem 4.1. Let $\lambda, \mu \in X$ and $r \geq 1$. If $\tilde{L}_{p^{r}}(\mu)$ is a composition factor of $\tilde{\Delta}_{r}(\lambda)$ then $\mu \uparrow \lambda$.

Here $\uparrow$ is the strong linkage relation, see [23], II.6.4:

Definition 4.2. Let $\lambda, \mu \in X$. We say that $\mu$ is strongly linked to $\lambda$ and write $\mu \uparrow \lambda$ iff there exist weights $\mu_{1}, \mu_{2}, \cdots, \mu_{r} \in X$ and reflections $s_{1}, s_{2}, \cdots, s_{r-1} \in W_{p}$ such that

$$
\mu_{i+1}=s_{i} \cdot \mu_{i} \text { and } \mu=\mu_{1} \leq \mu_{2} \leq \mu_{3} \leq \cdots \leq \mu_{r}=\lambda \text {. }
$$

Let now $M$ be an arbitrary finite dimensional $\bar{u}_{r} \bar{B}_{K}$-module (as always we assume $M$ is also a weight module, i.e. $M$ is of type $\mathbf{1}$ ). Then we set

$$
\bar{\Delta}_{p^{r}}^{\prime}(M)=\bar{U}_{K} \otimes_{\bar{u}_{r} \bar{B}_{K}} M .
$$

Clearly $\bar{\Delta}_{p^{r}}^{\prime}$ is an exact functor from the category of finite dimensional $\bar{u}_{r} \bar{B}_{K^{-}}$-modules into $\overline{\mathcal{O}}_{p}$. In addition it has the following properties.

If $V$ is a $\bar{B}_{K^{-}}$-module, then the Frobenius twist $V^{(r)}$ is a $\bar{u}_{r} \bar{B}_{K^{-}}$-module (with trivial $\bar{u}_{r^{-}}$ action) and we get [9], Lemma 3.2

$$
\bar{\Delta}_{p^{r}}^{\prime}\left(V^{(r)}\right) \simeq \bar{\Delta}_{p}(V)^{(r)} .
$$

If $L$ is a $\bar{U}_{K}$-module, then for all $\bar{u}_{r} \bar{B}_{K^{-}}$-modules $M$ we have the tensor identity

$$
\bar{\Delta}_{p^{r}}^{\prime}(L \otimes M) \simeq L \otimes \bar{\Delta}_{p^{r}}^{\prime}(M) .
$$

In particular, (4.1) and (4.2) show what happens, when we apply the $\bar{\Delta}_{p^{r}}^{\prime}$ to a simple $\bar{u}_{r} B_{K^{-}}$ module:

$$
\bar{\Delta}_{p^{r}}^{\prime}\left(\tilde{L}_{p^{r}}(\lambda)\right)=\bar{L}_{p}\left(\lambda^{0}\right) \otimes \bar{\Delta}_{p}\left(\lambda^{1}\right)^{(r)} \text { for all } \lambda \in X .
$$

Here $\lambda^{0} \in X_{p^{r}}$ and $\lambda^{1} \in X$ are as usual determined by the equation $\lambda=\lambda^{0}+p^{r} \lambda^{1}$.

Note also that

$$
\bar{\Delta}_{p^{r}}^{\prime} \tilde{\Delta}_{r}(\lambda)=\bar{\Delta}_{p}(\lambda)
$$


Applying $\bar{\Delta}_{p^{r}}^{\prime}$ to a composition series for the $\bar{u}_{r} B_{K^{-}}$module $\tilde{\Delta}_{r}(\lambda)$ we obtain by combining (4.3) and (4.4)

Proposition 4.3. Let $\lambda \in X$. Then $\bar{\Delta}_{p}(\lambda)$ has a filtration

$$
0=F_{0} \subset F_{1} \subset \cdots \subset F_{n}=\bar{\Delta}_{p}(\lambda)
$$

with $F_{i} / F_{i-1} \simeq \bar{L}_{p}\left(\mu_{i}^{0}\right) \otimes \bar{\Delta}_{p}\left(\mu_{i}^{1}\right)^{(r)}$ for some $\mu_{i} \in X, i=1,2, \cdots, n$.

We call a filtration as the one given in this theorem a $\left(p^{r}, \Delta\right)$-filtration. If $M$ has such a filtration then we denote by $\left(M: L_{p}\left(\mu^{0}\right) \otimes \bar{\Delta}_{p}\left(\mu^{1}\right)^{(r)}\right)$ the number of times a given quotient $\bar{L}_{p}\left(\mu^{0}\right) \otimes \bar{\Delta}_{p}\left(\mu^{1}\right)^{(r)}$ occurs in this filtration. The way we obtained the filtration of $\bar{\Delta}_{p}(\lambda)$ implies

Corollary 4.4. Let $\lambda, \mu \in X$. Then $\left(\bar{\Delta}_{p}(\lambda): \bar{L}_{p}\left(\mu^{0}\right) \otimes \bar{\Delta}_{p}\left(\mu^{1}\right)^{(r)}\right)=\left[\tilde{\Delta}_{r}(\lambda): \tilde{L}_{p^{r}}(\mu)\right]$.

Here we use the notation $\left[M: \tilde{L}_{p^{r}}(\mu)\right]$ for the composition factor multiplicity of $\tilde{L}_{p^{r}}(\mu)$ in a $\bar{u}_{r} \bar{B}_{K}$-module $M$.

Employing now also Theorem 4.1 we get the following linkage principle for $\left(p^{r}, \Delta\right)$-filtrations of Verma modules in $\overline{\mathcal{O}}_{p}$.

Theorem 4.5. Let $\lambda, \mu \in X$ and $r \geq 1$. If $\left(\bar{\Delta}_{p}(\lambda): \bar{L}_{p}\left(\mu^{0}\right) \otimes \bar{\Delta}_{p}\left(\mu^{1}\right)^{(r)}\right) \neq 0$ then $\mu \uparrow \lambda$.

4.2. Linkage principles for Verma modules in $\mathcal{O}_{q}$. We assume in this section that $q \in K$ has odd order $\ell>1$ (and $\ell$ is not divisible by 3 if $R$ contains a component of type $G_{2}$ ) and that $p>0$ (the $p=0$ case was treated in [7], Section 3). We can then argue just as in Section 4.1:

Let $M$ be a finite dimensional $u_{q} B_{q}$-module. As always we assume that $M$ is also a weight module. Then we define

$$
\Delta_{q}^{\prime}(M)=U_{q} \otimes_{u_{q} B_{q}} M
$$

If $M=N^{[\ell]}$ for some $\bar{U}_{K}$-module $N$ then we have, see [9] Lemma 3.2,

$$
\Delta_{q}^{\prime}\left(N^{[\ell]}\right) \simeq \bar{\Delta}_{p}(N)^{[\ell]}
$$

If $L$ is a $U_{q}$-module, then the tensor identity gives

$$
\Delta_{q}^{\prime}(L \otimes M) \simeq L \otimes \Delta_{q}^{\prime}(M) .
$$

Recall from Section 3.2 the definition of baby-Verma modules. Transitivity of induction gives

$$
\Delta_{q}^{\prime}\left(\tilde{\Delta}_{\ell}(\lambda)\right) \simeq \Delta_{q}(\lambda) \text { for all } \lambda \in X
$$

Consider the case where $M$ is irreducible, i.e. $M=\tilde{L}_{\ell}(\lambda)$ for some $\lambda$ in $X$. As usual we write $\lambda=\lambda^{0}+\ell \lambda^{1}$ with $\lambda^{0} \in X_{\ell}$ and $\lambda^{1} \in X$. Then $\tilde{L}_{\ell}(\lambda)=L_{q}\left(\lambda^{0}\right) \otimes \ell \lambda^{1}$. So by (4.6) and (4.5) we get

$$
\Delta_{q}^{\prime}\left(\tilde{L}_{\ell}(\lambda)\right) \simeq L_{q}\left(\lambda^{0}\right) \otimes \bar{\Delta}_{p}\left(\lambda^{1}\right)^{[\ell]}
$$

As $\tilde{L}_{\ell}(\lambda)$ is a quotient of $\tilde{\Delta}_{\ell}(\lambda)$ we have by (4.7) that $\Delta_{q}^{\prime}\left(\tilde{L}_{\ell}(\lambda)\right)$ is a quotient of $\Delta_{q}(\lambda)$. It follows that $\Delta_{q}^{\prime}(M) \in \mathcal{O}_{q}$ for all $M$.

Combining the above we obtain 
Proposition 4.6. Let $\lambda \in X$. Then $\Delta_{q}(\lambda)$ has a finite filtration in $\mathcal{O}_{q}$

$$
0=F_{0} \subset F_{1} \subset \cdots \subset F_{r}=\Delta_{q}(\lambda)
$$

with $F_{i} / F_{i-1} \simeq L_{q}\left(\mu_{i}^{0}\right) \otimes \bar{\Delta}_{p}\left(\mu_{i}^{1}\right)^{[\ell]}$ for some $\mu_{i} \in X, i=1,2, \cdots, n$.

A filtration like the one in this proposition is called an $(\ell, \Delta)$-filtration. Note that for any finite dimensional $u_{q} B_{q}$-module $M$ and any $\mu \in X$ the number of times the quotient $L_{q}\left(\mu^{0}\right) \otimes \bar{\Delta}_{p}\left(\mu^{1}\right)^{[\ell]}$ occurs in an $(\ell, \Delta)$-filtration of $\Delta_{q}^{\prime}(M)$ equals the multiplicity $\left[M: \tilde{L}_{\ell}(\mu)\right]$ of the composition factor $\tilde{L}_{\ell}(\mu)$ in $M$. Taking $M=\tilde{\Delta}_{\ell}(\lambda)$ we obtain

Corollary 4.7. Let $\lambda, \mu \in X$. Then

$$
\left(\Delta_{q}(\lambda): L_{q}\left(\mu^{0}\right) \otimes \bar{\Delta}_{p}\left(\mu^{1}\right)^{[\ell]}\right)=\left[\tilde{\Delta}_{\ell}(\lambda): \tilde{L}_{\ell}(\mu)\right]
$$

for all $\mu \in X$.

Just like in the modular case (Theorem 4.5 with $r=1$ ) we have

Theorem 4.8. Let $\lambda, \mu \in X$. If $\tilde{L}_{\ell}(\mu)$ is a composition factor of $\tilde{\Delta}_{\ell}(\lambda)$ then $\mu \uparrow \lambda$.

Of course the strong linkage relation here is with respect to $\ell$ instead of $p$.

This gives

Corollary 4.9. Let $\lambda, \mu \in X$. If $\left(\Delta_{q}(\lambda): L_{q}\left(\mu^{0}\right) \otimes \bar{\Delta}_{p}\left(\mu^{1}\right)^{[\ell]}\right) \neq 0$ then $\mu \uparrow \lambda$.

Consider now $M \in \mathcal{O}_{q}$ and assume $M$ has an $(\ell, \Delta)$-filtration. For each quotient $L_{q}\left(\mu^{0}\right) \otimes$ $\bar{\Delta}_{p}\left(\mu^{1}\right)^{[\ell]}$, which occurs in this filtration we choose now a $\left(p^{r}, \Delta\right)$-filtration of $\bar{\Delta}_{p}\left(\mu^{1}\right)$. This leads to a refined filtration of $M$ in which quotients have the form $L_{q}(\mu) \otimes \bar{L}_{p}(\nu)^{[\ell]} \otimes\left(\bar{\Delta}_{p}(\eta)^{(r)}\right)^{[\ell]}$ with $\mu \in X_{\ell}, \nu \in X_{p^{r}}$, and $\eta \in X$. We call such a filtration an $\left(\ell, p^{r}, \Delta\right)$-filtration of $M$.

Combining Corollary 4.3 and Corollary 4.6 we then get (in the obvious notation)

Corollary 4.10. Let $\lambda, \eta \in X$ and $\mu \in X_{\ell}, \nu \in X_{p^{r}}$. The Verma module $\Delta_{q}(\lambda)$ has an $\left(\ell, p^{r}, \Delta\right)$-filtration in which the multiplicities are given by

$$
\left(\Delta_{q}(\lambda): L_{q}(\mu) \otimes \bar{L}_{p}(\nu)^{[\ell]} \otimes\left(\bar{\Delta}_{p}(\eta)^{(r)}\right)^{[\ell]}\right)=\sum_{\zeta \in X}\left[\tilde{\Delta}_{\ell}(\lambda): \tilde{L}_{q}(\mu+\ell \zeta)\right]\left[\tilde{\Delta}_{r}(\zeta): \tilde{L}_{p^{r}}\left(\nu+p^{r} \eta\right)\right]
$$

(Note that this sum is finite.)

Remark 4.11. In the ordinary category $\mathcal{O}$ as well as in the characteristic zero category $\mathcal{O}_{q}$ we have duality functors, see [21, Section 3.3, respectively [7, Section 3.8. The very same recipe gives duality functors $D_{p}$ and $D_{q}$ on $\overline{\mathcal{O}}_{p}$ and $\mathcal{O}_{q}$. These functors preserve characters so we have in particular $D_{p}\left(\bar{L}_{p}(\lambda)\right) \simeq \bar{L}_{p}(\lambda)$ and $D_{q}\left(L_{q}(\lambda)\right) \simeq L_{q}(\lambda)$ for all $\lambda \in X$.

We shall denote the dual Verma modules by $\bar{\nabla}_{p}(\lambda)=D_{p}\left(\bar{\Delta}_{p}(\lambda)\right)$ and $\nabla_{q}(\lambda)=D_{q}\left(\Delta_{q}(\lambda)\right)$. Then all the above results have dual analogues. In particular, $\bar{\nabla}_{p}(\lambda)$ has for each $r$ a $\left(p^{r}, \nabla\right)$ filtration, and $\nabla_{q}(\lambda)$ has an $(\ell, \nabla)$-filtration satisfying the dual analogues of Theorem 4.4 and Corollary 4.8.

$$
\text { 5. } \mathfrak{g}=\mathfrak{s l}_{2}
$$

In this section we illustrate our results in the previous sections in the $\mathfrak{s l}_{2}$-case. 
5.1. The simple modules in $\overline{\mathcal{O}}_{p}=\overline{\mathcal{O}}_{p}\left(\mathfrak{s l}_{2}\right)$. We have $X=\mathbb{Z}$ and $X^{+}=\mathbb{Z}_{\geq 0}$. So the simple modules in $\overline{\mathcal{O}}_{p}$ are $\bar{L}_{p}(n)$ with $n \in \mathbb{Z}$, and among these the ones with $n \geq 0$ are the finite dimensional ones.

Let $V$ denote the 2-dimensional natural module for $\mathfrak{s l}_{2}$. Then it is well known that $\bar{L}_{p}(n)=$ $S^{n} V$ for $n=0,1, \cdots, p-1$, and we get

$$
\operatorname{ch} \bar{L}_{p}(n)=e^{n}+e^{n-2}+\cdots+e^{-n} \text { for all } n=0,1, \cdots, p-1 .
$$

Now let $n \geq 0$ be arbitrary and write $n=n_{0}+n_{1} p+\cdots+n_{r} p^{r}$ with $0 \leq n_{i} \leq p-1$ for all $i$. Then Theorem 3.6 says $\bar{L}_{p}(n) \simeq \bar{L}_{p}\left(n_{0}\right) \otimes \bar{L}_{p}\left(n_{1}\right)^{(1)} \otimes \cdots \otimes \bar{L}_{p}\left(n_{r}\right)^{(r)}$. This implies $\operatorname{dim} \bar{L}_{p}(n)=\left(n_{0}+1\right)\left(n_{1}+1\right) \cdots\left(n_{r}+1\right)$ and

$$
\operatorname{ch} \bar{L}_{p}(n)=\prod_{i=0}^{r}\left(e^{n_{i}}+e^{n_{i}-2}+\cdots+e^{-n_{i}}\right)^{(i)} .
$$

Now consider $n=-1$. An easy direct computation (alternatively use the arguments for (5.5) in the following section) shows that $\bar{L}_{p}(-1)=\bar{\Delta}_{p}(-1)$. Hence

$$
\operatorname{ch} \bar{L}_{p}(-1)=e^{-1}+e^{-3}+e^{-5}+\cdots .
$$

In this case Proposition 4.3 gives $\bar{\Delta}_{p}(-1) \simeq \overline{S t}_{r} \otimes \bar{\Delta}_{p}(-1)^{(r)}$ for all $r>0$.

Finally, consider $n<-1$. We choose $r$ so big that $p^{r}>-n$. Then $n$ has p-adic expansion $n=\left(p^{r}+n\right)+(-1) p^{r}$ and Theorem 3.6 implies $\bar{L}_{p}(n) \simeq \bar{L}\left(p^{r}+n\right) \otimes \bar{L}_{p}(-1)^{(r)}$. Therefore we get

$$
\operatorname{ch} \bar{L}_{p}(n)=\operatorname{ch} \bar{L}_{p}\left(p^{r}+n\right)\left(\operatorname{ch} \bar{L}_{p}(-1)\right)^{(r)} .
$$

Note that (5.2) and (5.3) determine the two characters on the right hand side.

5.2. The Verma modules in $\overline{\mathcal{O}}_{p}$. Let $n \in \mathbb{Z}$. Proposition 4.3 gives

(1) If $n \equiv-1(\bmod p)$ then $\bar{\Delta}_{p}(n) \simeq \overline{S t}_{1} \otimes \bar{\Delta}_{p}\left(\frac{n+1}{p}-1\right)^{(1)}$,

(2) if $n \not \equiv-1(\bmod p)$ we write $n=n_{0}+n_{1} p$ with $0 \leq n_{0}<p-1$ and get a short exact sequence

$$
0 \rightarrow \bar{L}_{p}\left(p-n_{0}-2\right) \otimes \bar{\Delta}_{p}\left(n_{1}-1\right)^{(1)} \rightarrow \bar{\Delta}_{p}(n) \rightarrow \bar{L}_{p}\left(n_{0}\right) \otimes \bar{\Delta}_{p}\left(n_{1}\right)^{(1)} \rightarrow 0 .
$$

This allows us to determine the structure of $\bar{\Delta}_{p}(n)$ for all $n$. For instance, we get

$$
\bar{\Delta}_{p}(-1)=\bar{L}_{p}(-1) \text {. }
$$

In fact, for $n=-1$ the isomorphism in (1) says $\bar{\Delta}_{p}(-1) \simeq \overline{S t}_{1} \otimes \bar{\Delta}_{p}(-1)^{(1)}$. Repeated use of (1) on the second tensor factor here leads to $\bar{\Delta}_{p}(-1) \simeq \overline{S t}_{r} \otimes \bar{\Delta}_{p}(-1)^{(r)}$. On the other hand, Steinberg's tensor product theorem, cf. Corollary 3.8, gives $\bar{L}_{p}(-1) \simeq S t_{r} \otimes \bar{L}_{p}(-1)^{(r)}$. This implies that the weight spaces of $\bar{\Delta}_{p}(-1)$ and $\bar{L}_{p}(-1)$ coincide.

When $n=0$ the short exact sequence (2) reads (noting that by Steinberg's tensor product theorem $\left.\bar{L}_{p}(p-2) \otimes \bar{L}_{p}(-1)^{(1)} \simeq \bar{L}_{p}(-2)\right)$

$$
0 \rightarrow \bar{L}_{p}(-2) \rightarrow \bar{\Delta}_{p}(0) \rightarrow \bar{\Delta}_{p}(0)^{(1)} \rightarrow 0 .
$$

Repeated use of this sequence shows that for any $r>0$ we have a short exact sequence

$$
0 \rightarrow M_{r} \rightarrow \bar{\Delta}_{p}(0) \rightarrow \Delta_{p}(0)^{(r)} \rightarrow 0,
$$


where the submodule $M_{r}$ has a finite composition series with factors $\bar{L}_{p}(-2), \bar{L}_{p}(-2)^{(1)}, \cdots, \bar{L}_{p}(-2)^{(r-1)}$. In particular, we see that $\bar{\Delta}_{p}(0)$ has simple socle equal to $\bar{L}_{p}(-2)$. In fact, similar computations give for general $n \geq 0$, that the socle of $\bar{\Delta}_{p}(n)$ is $\bar{L}_{p}(-n-2)$.

Likewise, we get for $n=-2$ the short exact sequence

$$
0 \rightarrow \bar{\Delta}_{p}(-2)^{(1)} \rightarrow \bar{\Delta}_{p}(-2) \rightarrow \bar{L}_{p}(-2) \rightarrow 0 .
$$

This leads for each $r>0$ to the following short exact sequences

$$
0 \rightarrow \bar{\Delta}_{p}(-2)^{(r)} \rightarrow \bar{\Delta}_{p}(-2) \rightarrow N_{r} \rightarrow 0,
$$

where the quotients $N_{r}$ have finite composition series with factors $\bar{L}_{p}(-2)^{(r-1)}, \bar{L}_{p}(-2)^{(r-2)}, \cdots$, $\bar{L}_{p}(-2)$. In particular, we see from this that $\operatorname{Hom}_{\overline{\mathcal{O}}_{p}}\left(\bar{L}_{p}(n), \bar{\Delta}_{p}(-2)\right)=0$ for all $n$. Again this result holds also when we replace -2 by any smaller integer, cf. Corollary 8.2 below for general $\mathfrak{g}$.

5.3. The simple modules in $\mathcal{O}_{q}=\mathcal{O}_{q}\left(\mathfrak{s l}_{2}\right)$. In this and the next section we consider the quantum $\mathfrak{s l}_{2}$-case where $q \in K$ is a primitive root of unity of order $\ell>1$. As always we assume for convenience that $\ell$ is odd.

We have analogues of the results in Section 5.1. Skipping further details we only state the following results (which determine all irreducible characters in $\mathcal{O}_{q}$ ):

$$
\operatorname{ch} L_{q}(n)=e^{n}+e^{n-2}+\cdots+e^{-n} \text { for } n=0,1, \cdots, \ell-1,
$$

$$
\operatorname{ch} L_{q}(n)=\operatorname{ch} L_{q}\left(n_{0}\right)\left(\operatorname{ch} \bar{L}_{p}\left(n_{1}\right)\right)^{(\ell)} \text { for all } n=n_{0}+n_{1} \ell \text { with } 0 \leq n_{0} \leq \ell-1, n_{1} \in \mathbb{Z},
$$

$$
L_{q}(-1)=\Delta_{q}(-1) \text { and } \operatorname{ch} L_{q}(-1)=e^{-1}+e^{-3}+e^{-5}+\cdots .
$$

Note that the two characters appearing on the right hand side in (5.10) are given by (5.9), respectively the formulas in Section [5.1.

5.4. The Verma modules in $\mathcal{O}_{q}$. We list the following results analogous to those in Section 5.2 .

There is an exact sequence

$$
0 \rightarrow L_{q}(-2) \rightarrow \Delta_{q}(0) \rightarrow \bar{\Delta}_{p}(0)^{[\ell]} \rightarrow 0 .
$$

Combining this with (5.7) we see that $\Delta_{q}(0)$ has for each $r \geq 0$ a submodule $M_{r}$ with composition factors $L_{q}(-2), L_{q}(-2 \ell), L_{q}(-2 \ell p), \cdots, L_{q}\left(-2 \ell p^{r-1}\right)$. The quotient of $M_{r}$ is $\left(\bar{\Delta}_{p}(0)^{(r)}\right)^{[\ell]}$. In particular, we see that $\bar{\Delta}_{p}(0)$ has simple socle. This fact remains true, when we replace 0 by any other non-negative integer, cf. Corollary 8.5 for general $\mathfrak{g}$.

Likewise, we have a short exact sequence

$$
0 \rightarrow \Delta_{q}(-2)^{[\ell]} \rightarrow \Delta_{q}(-2) \rightarrow L_{q}(-2) \rightarrow 0 .
$$

This leads for each $r \geq 0$ to a quotient $N_{r}=\Delta_{q}(-2) /\left(\bar{\Delta}_{p}(-2)^{(r)}\right)^{[\ell]}$ with composition factors $L_{q}\left(-2 \ell p^{r-1}\right), L_{q}\left(-2 \ell p^{r-2}\right), \cdots, \bar{L}_{q}(-2)$.

In particular, we see that $\operatorname{Hom}_{\mathcal{O}_{q}}\left(L_{q}(n), \Delta_{q}(-2)\right)=0$ for all $n$. This result holds also when we replace -2 by any smaller integer. 


\section{The special Verma module}

We return to the case of a general Cartan matrix, but continue to assume that $q$ is an $\ell^{\prime}$ th root of unity in a field $K$ of characteristic $p>0$. First we treat the case $\ell=1$.

\subsection{The special Verma module in $\overline{\mathcal{O}}_{p}$.}

Theorem 6.1. Let $\lambda \in X$. Then $\bar{\Delta}_{p}(\lambda)$ is irreducible if and only if $\lambda=-\rho$.

Proof. To prove that $\bar{\Delta}_{p}(-\rho)$ is irreducible we show that the surjection $\bar{\Delta}_{p}(-\rho) \rightarrow \bar{L}_{p}(-\rho)$ is an isomorphism. This follows once we check that

$$
\operatorname{dim}_{K} \bar{\Delta}_{p}(-\rho)_{-\rho-\mu} \leq \operatorname{dim}_{K} \bar{L}_{p}(-\rho)_{-\rho-\mu}
$$

for all $\mu \geq 0$. By the modular analogue of 2.1 the left hand side of 6.1 equals $P(\mu)$. By Corollary 3.8 we have $\bar{L}_{p}(-\rho) \simeq \overline{S t}_{r} \otimes \bar{L}_{p}(-\rho)^{(r)}$ for all $r>0$. Hence $\operatorname{dim}_{K} \bar{L}_{p}(-\rho)_{-\rho-\mu} \geq$ $\operatorname{dim}_{K}\left(\overline{S t}_{r}\right)_{\left(p^{r}-1\right) \rho-\mu}$. By the analoque of (3.1) this last dimension equals $\#\left\{\left(n_{\beta}\right)_{\beta \in R^{+}} \mid 0 \leq\right.$ $n_{\beta}<p^{r}$ for all $\beta$ and $\sum_{\beta \in R^{+}} n_{\beta} \beta=\mu$ \}. If $r$ is large enough then the conditions $n_{\beta}<p^{r}$ are automatically satisfied for all $\left(n_{\beta}\right)$ ) for which $\sum_{\beta} n_{\beta} \beta=\mu$. Hence this number equals $P(\mu)$ for all $r \gg 0$.

To prove the converse consider $\lambda \neq-\rho$ and write as usual $\lambda=\lambda^{0}+p^{r} \lambda^{1}$. Then there exists $r>0$ such that $\lambda^{0} \neq\left(p^{r}-1\right) \rho$ and by the analogue of Proposition 3.3 we have that $\tilde{\Delta}_{r}(\lambda)$ is not irreducible. Therefore the $\left(p^{r}, \Delta\right)$-filtration of $\bar{\Delta}_{p}(\lambda)$ from Proposition 4.3 contains more than one term and thus $\bar{\Delta}_{p}(\lambda)$ is not simple.

This result allows us in particular to give the following formula for the character of a simple module in $\overline{\mathcal{O}}_{p}$ with antidominant highest weight (recall from Section 2.3 the notation $\left.q^{-}=\sum_{\mu \leq-\rho} P(-\rho-\mu) e^{\mu}\right)$.

Corollary 6.2. Let $\lambda \in X^{-}$and choose $r$ so big that $p^{r} \rho+\lambda \in X_{r}$. Then

$$
\operatorname{ch} \bar{L}_{p}(\lambda)=\operatorname{ch} \bar{L}_{p}\left(p^{r} \rho+\lambda\right)\left(q^{-}\right)^{(r)} .
$$

Proof. When we combine Theorem 3.6 with Theorem 6.1 we get $\bar{L}_{p}(\lambda) \simeq \bar{L}_{p}\left(p^{r} \rho+\lambda\right) \otimes$ $\bar{\Delta}_{p}(-\rho)^{(r)}$. The formula then follows from (2.1).

The special Verma module $\bar{\Delta}_{p}(-\rho)$ has also other nice properties:

Theorem 6.3. The Verma module $\bar{\Delta}_{p}(-\rho)$ is projective and injective in $\overline{\mathcal{O}}_{p}$.

Proof. Recall that since $\bar{\Delta}_{p}(-\rho)$ is simple, it is self-dual. Hence it is enough to check that it is projective.

Suppose $M \rightarrow N$ is a surjection in $\overline{\mathcal{O}}_{p}$. We claim that the induced map

$$
\operatorname{Hom}_{\overline{\mathcal{O}}_{p}}\left(\bar{\Delta}_{p}(-\rho), M\right) \rightarrow \operatorname{Hom}_{\overline{\mathcal{O}}_{p}}\left(\bar{\Delta}_{p}(-\rho), N\right)
$$

is also surjective.

The definition of Verma modules implies that for any $V \in \overline{\mathcal{O}}_{p}$ we have

$$
\operatorname{Hom}_{\overline{\mathcal{O}}_{p}}\left(\bar{\Delta}_{p}(-\rho), V\right) \simeq\left\{v \in V_{-\rho} \mid \bar{B}_{K} v=K v\right\} .
$$


To check the surjectivity of (6.2) we consider first the special case, where $M$ has no weights strictly bigger than $-\rho$. In that case (6.3) gives $\operatorname{Hom}_{\overline{\mathcal{O}}_{p}}\left(\bar{\Delta}_{p}(-\rho), M\right)=M_{-\rho}$ and $\operatorname{Hom}_{\overline{\mathcal{O}}_{p}}\left(\bar{\Delta}_{p}(-\rho), N\right)=N_{-\rho}$. Hence in this case the claim certainly holds.

So consider now a general $M \in \overline{\mathcal{O}}_{p}$. We can find weights $\lambda_{1}, \lambda_{2}, \cdots, \lambda_{n}$ such that all weights $\mu$ of $M$ satisfy $\mu \leq \lambda_{i}$ for some $i$. By Theorem 6.1 and Theorem [3.6 we have $\bar{\Delta}_{p}(-\rho)=$ $S t_{r} \otimes \bar{\Delta}_{p}(-\rho)^{(r)}$. By Proposition 3.16 (2) this gives

$$
\operatorname{Hom}_{\overline{\mathcal{O}}_{p}}\left(\bar{\Delta}_{p}(-\rho), M\right) \simeq \operatorname{Hom}_{\overline{\mathcal{O}}_{p}}\left(\bar{\Delta}_{p}(-\rho), \operatorname{Hom}_{\bar{u}_{r}}\left(S t_{r}, M\right)^{(-r)}\right)
$$

Now the natural map $S t_{r} \otimes \operatorname{Hom}_{\bar{u}_{r}}\left(S t_{r}, M\right) \rightarrow M$ is injective and hence for any weight $\mu$ of $\operatorname{Hom}_{\bar{u}_{r}}\left(S t_{r}, M\right)^{(-r)}$ we must have that $\left(p^{r}-1\right) \rho+p^{r} \mu$ is a weight of $M$. This implies that $\left(p^{r}-1\right) \rho+p^{r} \mu \leq \lambda_{i}$ for some $i$. So if $r$ is large enough we cannot have $\mu$ strictly bigger than $-\rho$. Combining this with (6.4) we then deduce the general case from the special case treated above.

\subsection{The special Verma module in $\mathcal{O}_{q}$. In analogy with Theorem 6.1 we get}

Theorem 6.4. Let $\lambda \in X$. Then $\Delta_{q}(\lambda)$ is irreducible if and only if $\lambda=-\rho$.

Proof. As $\tilde{\Delta}_{q}((\ell-1) \rho)=S t_{q}$ we see from Proposition 4.6 that $\Delta_{q}(-\rho) \simeq S t_{q} \otimes \bar{\Delta}_{p}(-\rho)^{[\ell]}$. By Theorem 3.4 we get on the other hand $L_{q}(-\rho) \simeq S t_{q} \otimes \bar{L}_{p}(-\rho)^{[\ell]}$. Hence it follows from Theorem 6.1 that $\Delta_{q}(-\rho)$ is irreducible.

The proof of the converse is completely analogous to the proof given in Theorem 6.1 ,

Theorem 6.5. The Verma module $\Delta_{q}(-\rho)$ is projective and injective in $\mathcal{O}_{q}$.

Proof. As in the proof of Theorem 6.4 we have $\Delta_{q}(-\rho)=S t_{q} \otimes \bar{\Delta}_{p}(-\rho)^{[\ell]}$. Therefore, by Proposition 3.16 (1) we get

$$
\operatorname{Hom}_{\mathcal{O}_{q}}\left(\Delta_{q}(-\rho), M\right) \simeq \operatorname{Hom}_{\overline{\mathcal{O}}_{p}}\left(\bar{\Delta}_{p}(-\rho), \operatorname{Hom}_{u_{q}}\left(S t_{q}, M\right)^{[-\ell]}\right)
$$

for all $M \in \mathcal{O}_{q}$. Now $\operatorname{Hom}_{u_{q}}\left(S t_{q},-\right)$ is exact (e.g. by the $q$-analogue of [23], Lemma II.11.8), and according to Theorem 6.3 so is $\operatorname{Hom}_{\overline{\mathcal{O}}_{p}}\left(\bar{\Delta}_{p}(-\rho),-\right)$.

\section{Tilting MOdules}

We have both finite dimensional tilting modules and infinite dimensional ones in our BGGcategories. First we consider the latter class, which we shall call $\infty$-tilting modules. We prove their most basic properties. Then we recall a few facts about the better studied class of finite dimensional tilting modules. We show how these facts (under some restrictions on $p$ ) make us able to obtain an explicit relation between the indecomposable $\infty$-tilting modules and certain indecomposable finite dimensional tilting modules.

7.1. $\infty$-tilting modules. Our results in this section hold true in $\mathcal{O}_{q}$ as well as in $\overline{\mathcal{O}}_{p}$ and the proofs are completely analogous. Therefore we formulate and prove our statements only in the first case.

Recall from Remark 4.11 that we have a duality functor $D_{q}$ on $\mathcal{O}_{q}$ (as well as a similar duality $D_{p}$ on $\left.\overline{\mathcal{O}}_{p}\right)$, and that for each $\lambda \in X$ we define $\nabla_{q}(\lambda)=D_{q}\left(\Delta_{q}(\lambda)\right)$. 
Definition 7.1. A module $Q$ in $\mathcal{O}_{q}$ is called an $\infty$-tilting module if it has a finite $\Delta$-filtration as well as a finite $\nabla$-filtration.

We emphasize that in this paper by $\Delta$-filtration we mean a filtration in which each successive quotient is a Verma module. If we replace Verma by Weyl then we get the finite dimensional tilting modules instead.

Example 7.2. We have already come across one $\infty$-tilting module, namely the special Verma module $\Delta_{q}(-\rho)$, which we studied in the previous section. By Theorem 6.4 we have $\left.\Delta_{q}(-\rho)\right) \simeq$ $L_{q}(-\rho) \simeq \nabla_{q}(-\rho)$.

If $Q$ has a finite $\Delta$-filtration we shall denote the multiplicity with which a given Verma module $\Delta_{q}(\lambda)$ occurs as a quotient in such a filtration by $\left(Q: \Delta_{q}(\lambda)\right)$. Note that the head of $Q$ is contained in $\oplus L_{q}(\lambda)$ with $\lambda$ occurring with multiplicity $\left(Q: \Delta_{q}(\lambda)\right)$ in the sum. In particular, the head of $Q$ consists of finitely many simple modules. It follows that $Q$ splits into a finite number of indecomposable summands. Each of these summands have a $\Delta$-filtration. Hence if $Q$ is an $\infty$-tilting module it follows that it splits into a finite number of indecomposable $\infty$-tilting modules.

Lemma 7.3. Suppose $Q$ has a finite $\Delta$-filtration and let $F$ be a finite dimensional module. Then $F \otimes Q$ has a finite $\Delta$-filtration, and $\left(F \otimes Q: \Delta_{q}(\lambda)\right)=\sum_{\mu}\left(Q: \Delta_{q}(\mu)\right) \operatorname{dim}_{K} F_{\lambda-\mu}$ for all $\lambda$.

Proof. It is clearly enough to check that $F \otimes \Delta_{q}(\lambda)$ has a $\Delta$-filtration for all $\lambda \in X$. This is seen by the standard argument: Observe that $F \otimes \Delta_{q}(\lambda) \simeq \Delta_{q}(F \otimes \lambda)$. Now take a $B_{q}$-filtration of $F$ with 1-dimensional quotients, tensor this filtration by $\lambda$ and apply the exact functor $\Delta_{q}$ to get a $\Delta$-filtration of $F \otimes \Delta_{q}(\lambda)$. This construction also proves the multiplicity formula.

Remark 7.4. It follows immediately from this lemma that if $Q$ is $\infty$-tilting and $F$ is a finite dimensional self-dual module then $Q \otimes F$ is also $\infty$-tilting.

Theorem 7.5. If $\lambda \in X^{+}-\rho$ then there exists a unique (up to isomorphism) indecomposable $\infty$-tilting module $T_{q}^{\infty}(\lambda)$ in $\mathcal{O}_{q}$ with $T_{q}^{\infty}(\lambda)_{\lambda} \simeq K$ and $T_{q}^{\infty}(\lambda)_{\mu} \neq 0$ if and only if $\mu \leq \lambda$.

Proof. Let $\lambda \in X^{+}-\rho$. Then $\lambda+\rho \in X^{+}$and hence $L_{q}(\lambda+\rho)$ is finite dimensional. When we combine Lemma 7.3 (and Remark 7.4) with Theorem 6.4 we get that $L_{q}(\lambda+\rho) \otimes \Delta_{q}(-\rho)$ is $\infty$-tilting. Moreover, $L_{q}(\lambda+\rho) \otimes \Delta_{q}(-\rho)$ has a unique indecomposable summand with highest weight $\lambda$ because $\lambda$ is the maximal weight of $L_{q}(\lambda+\rho) \otimes \Delta_{q}(-\rho)$ and it occurs with multiplicity 1. So we define $T_{q}^{\infty}(\lambda)$ to be that summand and have thus proved the existence part of the theorem.

To establish the uniqueness of $T_{q}^{\infty}(\lambda)$ we first observe that since $\Delta_{q}(-\rho)$ is projective and injective in $\mathcal{O}_{q}$ (according to Theorem 6.5) so is $T_{q}^{\infty}(\lambda)$. Now assume that $Q$ is another indecomposable tilting module in $\mathcal{O}_{q}$ with highest weight $\lambda$. Then both $T_{q}^{\infty}(\lambda)$ and $Q$ projects onto $\nabla_{q}(\lambda)$, and therefore there is a homomorphism $\phi: T_{q}^{\infty}(\lambda) \rightarrow Q$ which is non-zero on the $\lambda$ weight space. Likewise, $\Delta_{q}(\lambda)$ has inclusions into both $T_{q}^{\infty}(\lambda)$ and $Q$. The injectivity of $T_{q}^{\infty}(\lambda)$ gives a homomorphism $\psi: Q \rightarrow T_{q}^{\infty}(\lambda)$ which is also non-zero on the $\lambda$ weight space. We claim that the composite $\psi \circ \phi$ is an isomorphism so that $T_{q}^{\infty}(\lambda)$ is a summand of $Q$ (and hence isomorphic to $Q$ ). 
By construction $\psi \circ \phi$ acts as a non-zero scalar $a$ on the $\lambda$ weight space. We will prove that $f=\psi \circ \phi-a \operatorname{Id}_{T_{q}^{\infty}(\lambda)}$ is nilpotent. This implies that $\psi \circ \phi$ is an isomorphism.

For each $\mu \in X$ we denote by $f_{\mu}$ the restriction of $f$ to $T_{q}^{\infty}(\lambda)_{\mu}$. Then $f_{\lambda}=0$ so that $f$ is certainly not an isomorphism. For any $\mu$ we have that $f_{\mu}$ is an endomorphism of a finite dimensional vector space and therefore the sequence $\operatorname{Im} f_{\mu} \supset \operatorname{Im} f_{\mu}^{2} \supset \cdots$ must stablize, i.e. there exists $n(\mu) \in \mathbb{Z}_{>0}$ such that $\operatorname{Im} f_{\mu}^{n(\mu)}=\operatorname{Im} f_{\mu}^{n(\mu)+1}=\cdots$. We denote now by $\mu_{1}, \mu_{2}, \cdots \mu_{r}$ the highest weights of the Verma modules that occur in a $\Delta$-filtration of $T_{q}^{\infty}(\lambda)$ and set $n=\max \left\{n\left(\mu_{i}\right) \mid i=1,2, \cdots r\right\}$. The usual fitting lemma argument then shows that $T_{q}^{\infty}(\lambda)_{\mu_{i}}=$ $\operatorname{Im} f_{\mu_{i}}^{n} \oplus \operatorname{Ker} f_{\mu_{i}}^{n}$ for all $i$. This implies that the submodule $M=\operatorname{Im} f^{n} \cap \operatorname{Ker} f^{n}$ of $T_{q}^{\infty}(\lambda)$ is 0 . In fact, by the choice of $n$ we have $M_{\mu_{i}}=0$ for all $\mathrm{i}$, and hence $\operatorname{Hom}_{\mathcal{O}_{q}}\left(M, \nabla_{q}\left(\mu_{i}\right)\right)=0$ for all $i$. We conclude that $\operatorname{Hom}_{\mathcal{O}_{q}}\left(M, T_{q}^{\infty}(\lambda)\right)=0$, i.e $M=0$.

Using the injectivity of $T_{q}^{\infty}(\lambda)$ we get likewise that $M^{\prime}=T_{q}^{\infty}(\lambda) /\left(\operatorname{Im} f^{n}+\operatorname{Ker} f^{n}\right)$ is 0 , because $\operatorname{Hom}_{\mathcal{O}_{q}}\left(\Delta_{q}\left(\mu_{i}\right), M^{\prime}\right)=0$ for all $i$. We conclude that $T_{q}^{\infty}(\lambda)=\operatorname{Im} f^{n} \oplus \operatorname{Ker} f^{n}$. As $T_{q}^{\infty}(\lambda)$ is indecomposable it follows that $f^{n}=0$.

Remark 7.6. Note that we cannot expect to extend the result in this theorem to all $\lambda \in X$. Already for $\mathfrak{s} l_{2}$ the result fails for $\lambda=n<-1$. In fact, if all weights of a module in $\mathcal{O}_{q}\left(\mathfrak{s l}_{2}\right)$ are less than -1 then it cannot be $\infty$-tilting, because no Verma module $\Delta_{q}(n)$ with $n<-1$ has a socle, see Section 5 .

7.2. Finite dimensional tilting modules. We recall some key facts about finite dimensional tilting modules in $\overline{\mathcal{O}}_{p}$ and $\mathcal{O}_{q}$. In the case $\overline{\mathcal{O}}_{p}$ this class identifies with the tilting modules for the almost simple algebraic group corresponding to $R$. These are well-studied, see e.g. [14, [23], Section II.E. In the quantum case the analogous facts hold, see [2] and [4].

Let $h$ denote the Coxeter number for $R$.

(1) For each $\mu \in X^{+}$there exists a unique (up to isomorphisms) indecomposable finite dimensional tilting module $\bar{T}_{p}(\mu)$ with weights $\leq \mu$ and with $\operatorname{dim}_{K} \bar{T}_{p}(\mu)_{\mu}=1$, [23], II.E.6.

(2) If $r>0$ and $\mu \in X_{r}$ then $\overline{S t}_{r} \otimes \bar{T}_{p}(\mu)$ is a tilting module with indecomposable summands having highest weights in $\left\{\nu \in\left(p^{r}-1\right) \rho+X^{+} \mid \nu \leq\left(p^{r}-1\right) \rho+\mu\right\}$, [23], II.E.8

(3) Suppose $p \geq 2 h-2$. If $\lambda \in X_{r}$ we set $\hat{\lambda}=2\left(p^{r}-1\right) \rho+w_{0} \lambda$. Then $\bar{T}_{p}(\hat{\lambda})_{\left.\right|_{\bar{u}_{1}}}$ is an indecomposable projective (and injective) $\bar{u}_{1}$-module. Its head and socle are isomorpic to $\bar{L}_{p}(\lambda)_{\left.\right|_{\bar{u}_{1}}}$, [23], II.E.9.

(4) For each $\mu \in X^{+}$there exists a unique (up to isomorphisms) indecomposable finite dimensional tilting module $T_{q}(\mu)$ with weights $\leq \mu$ and $\operatorname{with}^{\operatorname{dim}_{K}} T_{q}(\mu)_{\mu}=1$, [2, Section 4.

(5) Note that $S t_{q}=L_{q}((\ell-1) \rho)$ is tilting, i.e. $S t_{q}=T_{q}((\ell-1) \rho)$. If $\mu \in X_{\ell}$ then $S t_{q} \otimes T_{q}(\mu)$ is a finite dimensional tilting module with indecomposable summands $T_{q}(\nu)$, where $\nu \in(\ell-1) \rho+X^{+}$and $\nu \leq(\ell-1) \rho+\mu$, [4], Section 4 .

(6) Suppose $p \geq 2 h-2$. If $\lambda \in X_{\ell}$ and $\hat{\lambda}=2(\ell-1) \rho+w_{0} \lambda$ then $T_{q}(\hat{\lambda})_{\left.\right|_{\bar{u}_{q}}}$ is an indecomposable projective (and injective) $\bar{u}_{q}$-module. Its head and socle are isomorpic to $L_{q}(\lambda)_{\left.\right|_{\bar{u}_{q}}}$, [5], Proposition 5.7.

We now prove how (1) - (3) lead to the following result. 
Theorem 7.7. Let $\lambda \in X^{+}-\rho$ and take $r$ so big that $\lambda+\rho \in X_{p^{r}}$. Then we have

(1) $\bar{T}_{p}\left(\lambda+p^{r} \rho\right) \otimes \bar{\Delta}_{p}(-\rho)^{(r)}$ is $\infty$-tilting,

(2) Suppose $p \geq 2 h-2$. Then $\bar{T}_{p}^{\infty}(\lambda) \simeq \bar{T}_{p}\left(\lambda+p^{r} \rho\right) \otimes \bar{\Delta}_{p}(-\rho)^{(r)}$.

Proof. By (2) above we have that $\bar{T}_{p}\left(\lambda+p^{r} \rho\right)$ is a summand of $\bar{T}_{p}(\lambda+\rho) \otimes \overline{S t}_{r}$. Hence using Theorem 3.6 we get that $\bar{T}_{p}\left(\lambda+p^{r} \rho\right) \otimes \bar{\Delta}_{p}(-\rho)^{(r)}$ is a summand of $\bar{T}_{p}(\lambda+\rho) \otimes \bar{\Delta}_{p}(-\rho)$ and the first part of the theorem follows.

Under the assumption $p \geq 2 h-2$ we have from (3) above that $\bar{T}_{p}\left(\lambda+p^{r} \rho\right)$ has simple socle as $\bar{u}_{r}$-module. Therefore $\bar{T}_{p}\left(\lambda+p^{r} \rho\right) \otimes \bar{\Delta}_{p}(-\rho)^{(r)}$ has simple $\bar{U}_{K^{-}}$-socle. Hence it is in particular indecomposable. Since it has highest weight $\lambda$ it is therefore isomorphic to $\bar{T}_{p}^{\infty}(\lambda)$.

Remark 7.8. S. Donkin has conjectured that the assumption $p \geq 2 h-2$ in (3) above is unneccessary. However, Bendel, Nakano, Pillen and Sobaje found a counterexample (for $p=2$ and $R$ of type $G_{2}$ ) in [11]. In a recent preprint [12] the same authors have verified Donkin's conjecture in several low rank cases and announced further counter examples for $p=2$ (type $B_{3}$ ) and $p=3$ (type $C_{3}$ ).

Whenever, the conjecture holds we can omit the assumption on $p$ in Theorem 7.7 (2) as well as in Theorem 7.9 (2) below. - See also [12] for some background on Donkin's conjecture.

Next we shall prove that (4) - (6) give analogous results in $\mathcal{O}_{q}$.

Theorem 7.9. Let $\lambda \in X^{+}-\rho$ and take $r$ so big that $\lambda+\rho \in X_{\ell p^{r}}$. Then

(1) $T_{q}\left(\lambda+\ell p^{r} \rho\right) \otimes\left(\bar{\Delta}_{p}(-\rho)^{(r)}\right)^{[\ell]}$ is $\infty$-tilting,

(2) Suppose $p \geq 2 h-2$. Then $T_{q}^{\infty}(\lambda) \simeq T_{q}\left(\lambda+\ell p^{r} \rho\right) \otimes\left(\bar{\Delta}_{p}(-\rho)^{(r)}\right)^{[\ell]}$.

Proof. This proof runs parallel to the proof of Theorem 7.7 Note that $\lambda+\rho \in X^{+}$and that $\lambda+\ell p^{r} \rho$ is the maximal weight of $T_{q}(\lambda+\rho) \otimes S t_{q} \otimes \overline{S t}_{r}^{[\ell]}$. This implies that the $\infty$-tilting module $T_{q}(\lambda+\rho) \otimes \bar{\Delta}_{q}(-\rho) \simeq T_{q}(\lambda+\rho) \otimes S t_{q} \otimes\left(\overline{S t}_{r} \otimes \bar{\Delta}_{p}(-\rho)^{(r)}\right)^{[\ell]}$ contains $T_{q}\left(\lambda+\ell p^{r} \rho\right) \otimes\left(\bar{\Delta}_{p}(-\rho)^{(r)}\right)^{[\ell]}$ as a summand. Hence the first part of the theorem holds.

To check the second part it is enough to verify that $T_{q}\left(\lambda+\ell p^{r} \rho\right) \otimes\left(\bar{\Delta}_{p}(-\rho)^{(r)}\right)^{[\ell]}$ has simple socle. This follows from (6) and (3) above combined with [5], Corollary 5.8.

\section{Projective And injective modules}

We preserve the assumptions on $\ell$ and $p$ from the previous sections. So far we have found one projective module (namely the special Verma module) in each of the categories $\overline{\mathcal{O}}_{p}$ and $\mathcal{O}_{q}$. In the present section we use this projective module to obtain projective covers and injective envelopes of all simple modules with antidominant highest weights. We also show that the same cannot be done when the highest weight is not antidominant.

It turns out that the projective covers we obtain are also indecomposable $\infty$-tilting modules. This leads to a reciprocity law relating the Verma multiplicities in indecomposable $\infty$-tilting modules to multiplicities in the $\left(p^{r}, \Delta\right)$-, respectively $\left(\ell, p^{r}, \Delta\right)$-filtration of the Verma modules. 
8.1. Projectives and injectives in $\overline{\mathcal{O}}_{p}$. Set $X^{-}=\left\{\lambda \in X \mid\left\langle\lambda, \alpha^{\vee}\right\rangle \leq-1\right.$ for all $\left.\alpha \in S\right\}$. This is the set of antidominant weights or, more precisely, the closure of the antidominant cone in $X$.

Theorem 8.1. For each $\lambda \in X^{-}$there exists a projective indecomposable module $\bar{P}_{p}(\lambda) \in \overline{\mathcal{O}}_{p}$ with head $\bar{L}_{p}(\lambda)$. Moreover, $\bar{P}_{p}(\lambda)$ is also the injective envelope of $\bar{L}_{p}(\lambda)$ and coincides with the $\infty$-tilting module $\bar{T}_{p}^{\infty}\left(w_{0} \cdot \lambda\right)$.

Proof. Let $\lambda \in X^{-}$. Then we define

$$
\bar{P}_{p}(\lambda)=\bar{T}_{p}^{\infty}\left(w_{0} \cdot \lambda\right) .
$$

The theorem follows once we prove that $\bar{T}_{p}^{\infty}\left(w_{0} \cdot \lambda\right)$ is projective and has $\bar{L}_{p}(\lambda)$ as a quotient.

By construction, see the proof of Theorem 7.5, $\bar{T}_{p}^{\infty}\left(w_{0} \cdot \lambda\right)$ is a summand of $\bar{L}_{p}\left(w_{0}(\lambda+\rho)\right) \otimes$ $\bar{\Delta}_{p}(-\rho)$. Now Theorem 6.3 says that $\bar{\Delta}_{p}(-\rho)$ is projective and hence so are $\bar{L}_{p}\left(w_{0}(\lambda+\rho)\right) \otimes$ $\bar{\Delta}_{p}(-\rho)$ and its summand $\bar{T}_{p}^{\infty}\left(w_{0} \cdot \lambda\right)$.

We claim that $\bar{L}_{p}(\lambda)$ is a submodule of $\bar{T}_{p}^{\infty}\left(w_{0} \cdot \lambda\right)$. As $\bar{T}_{p}^{\infty}\left(w_{0} \cdot \lambda\right)$ is selfdual this is equivalent to $\bar{L}_{p}(\lambda)$ being a quotient. Now the highest weight of $\bar{T}_{p}^{\infty}\left(w_{0} \cdot \lambda\right)$ is $w_{0} \cdot \lambda$ and therefore $\bar{\Delta}_{p}\left(w_{0} \cdot \lambda\right)$ is a submodule. It thus suffices to prove that $\bar{L}_{p}(\lambda) \subset \bar{\Delta}_{p}\left(w_{0} \cdot \lambda\right)$. However, if we choose $r$ so large that $w_{0} \cdot \lambda \in X_{p^{r}}$ then $\tilde{L}_{p^{r}}(\lambda)$ is the socle of $\tilde{\Delta}_{r}\left(w_{0} \cdot \lambda\right)$, see [23, Proposition II.9.6. It follows that $\bar{L}\left(\lambda+p^{r} \rho\right) \otimes \bar{\Delta}(-\rho)^{(r)}$ is the first term in a $\left(p^{r}, \Delta\right)$ filtration of $\bar{\Delta}_{p}\left(w_{0} \cdot \lambda\right)$, see Proposition 4.3. However, by Theorem 4.5 and Theorem 6.1 we have $\bar{L}_{p}\left(\lambda+p^{r} \rho\right) \otimes \bar{\Delta}_{p}(-\rho)^{(r)} \simeq \bar{L}_{p}(\lambda)$.

Corollary 8.2. If $\lambda \in-\rho+X^{+}$then the socle of $\bar{\Delta}_{p}(\lambda)$ equals $\bar{L}_{p}\left(w_{0} \cdot \lambda\right)$.

Proof. As in the proof of Theorem 8.1 we get that $\bar{\Delta}_{p}(\lambda)$ is a submodule of $\bar{P}_{p}\left(w_{0} \cdot \lambda\right)$.

We shall now show that the analogue of Theorem 8.1 fails for all irreducible modules with highest weight outside $X^{-}$.

Theorem 8.3. If $P$ is a projective module in $\overline{\mathcal{O}}_{p}$ then $\operatorname{Hom}_{\overline{\mathcal{O}_{p}}}\left(P, \bar{L}_{p}(\lambda)\right)=0$ for all $\lambda \in X \backslash X^{-}$. Likewise, when $\lambda \notin X^{-}$an injective module $I$ in $\overline{\mathcal{O}}_{p}$ cannot contain $\bar{L}_{p}(\lambda)$.

Proof. Suppose that $P$ is a projective module in $\overline{\mathcal{O}}_{p}$. If there is a non-zero map $P \rightarrow \bar{L}_{p}(\lambda)$ for some $\lambda \in X$, then there is also for each $r \geq 1$ a non-zero map $P \rightarrow \bar{L}_{p}\left(\lambda^{0}\right) \otimes \bar{\nabla}_{p}\left(\lambda^{1}\right)^{(r)}$. Set $\hat{\lambda}=2\left(p^{r}-1\right) \rho+w_{0} \lambda^{0}+p^{r} \lambda^{1}$. Then $\tilde{L}_{p^{r}}(\lambda)$ is the head of $\tilde{\nabla}_{p^{r}}(\hat{\lambda})$, see [23], Proposition II.9.6, and we get that the $\left(p^{r}, \nabla\right)$-filtration of $\bar{\nabla}_{p}(\hat{\lambda})$ constructed in Proposition 4.3 contains a surjection $\bar{\nabla}_{p}(\hat{\lambda}) \rightarrow \bar{L}_{p}\left(\lambda^{0}\right) \otimes \bar{\nabla}_{p}\left(\lambda^{1}\right)^{(r)}$. As $P$ is projective this results in a non-zero map $P \rightarrow \bar{\nabla}_{p}(\hat{\lambda})$. Therefore $\hat{\lambda}$ must be a weight of $P$. We shall now prove that this is impossible if $\lambda \notin X^{-}$and $r \gg 0$.

As we are going to vary the exponent of $p$ we introduce the following notation (for use in the rest of this proof)

$$
\begin{gathered}
\lambda=\lambda^{0}(r)+p^{r} \lambda^{1}(r) \text { where } \lambda^{0}(r) \in X_{r} \text { and } \lambda^{1}(r) \in X . \\
\hat{\lambda}(r)=\widehat{\lambda^{0}(r)}+p^{r} \lambda^{1}(r) \text { where } \widehat{\lambda^{0}(r)}=2\left(p^{r}-1\right) \rho+w_{0} \lambda^{0}(r)
\end{gathered}
$$


Suppose now $\lambda \in X \backslash X^{-}$. Then there exists $\alpha \in S$ such that $\left\langle\lambda, \alpha^{\vee}\right\rangle \geq 0$. Choose $r$ so big that $\omega=\lambda^{1}(r)$ satisfies $\left\langle\omega, \beta^{\vee}\right\rangle \in\{-1,0\}$ for all $\beta \in S$. We have $\left\langle\omega, \alpha^{\vee}\right\rangle=0$. Note also that $\omega$ is equal to $\lambda^{1}\left(r^{\prime}\right)$ for all $r^{\prime} \geq r$.

We have $\omega^{0}(s)=\left(p^{s}-1\right)(-\omega)$ and $\omega^{1}(s)=\omega$. It follows that

$\lambda^{0}(r+s)=\lambda^{0}(r)+p^{r}\left(1-p^{s}\right) \omega$ and $\lambda \widehat{(r+s)}=2\left(p^{r+s}-1\right) \rho+w_{0} \lambda^{0}(r)+p^{r}\left(1-p^{s}\right) w_{0}(\omega)+p^{r+s} \omega$.

We get from this

$$
\left\langle\hat{\lambda}(r+s), \alpha^{\vee}\right\rangle \geq 2\left(p^{r+s}-1\right)-\left(p^{r}-1\right)+p^{r}\left(1-p^{s}\right)=p^{r+s}-1,
$$

and

$$
\left\langle\hat{\lambda}(r+s), \beta^{\vee}\right\rangle \geq 2\left(p^{r+s}-1\right)-\left(p^{r}-1\right)+p^{r}\left(1-p^{s}\right)-p^{r+s}=-1 \text { for all } \beta \in S \backslash\{\alpha\} .
$$

The inequalities (8.2) and (8.3) show that $\hat{\lambda}(r+s)+\rho \in X^{+}$for all $s$. Now since $P$ is a module in $\overline{\mathcal{O}}_{p}$ there exist $\mu_{1}, \mu_{2}, \cdots, \mu_{n}$ such that for any weight $\nu$ of $P$ we have $\nu \leq \mu_{i}$ for some $i$. But suppose $\hat{\lambda}(r+s) \leq \mu_{i}$. We have

$$
\mu_{i}=\sum_{\beta \in S} a_{\beta} \beta, \rho=\sum_{\beta \in S} b_{\beta} \beta, \text { and } \hat{\lambda}(r+s)+\rho=\sum_{\beta \in S} c_{\beta}(s) \beta
$$

for some $a_{\beta} \in \mathbb{Q}, b_{\beta} \in \mathbb{Q}_{>0}$ and $c_{\beta}(s) \in \mathbb{Q}_{\geq 0}$. Then the inequality $\hat{\lambda}(r+s) \leq \mu_{i}$ is equivalent to

$$
c_{\beta}(s)-b_{\beta} \leq a_{\beta} \text { for all } \beta \in S .
$$

Combining this inequality for $\beta=\alpha$ with (8.2) we get

$$
p^{r+s} \leq\left\langle\hat{\lambda}(r+s)+\rho, \alpha^{\vee}\right\rangle \leq 2 c_{\alpha}(s) \leq 2\left(a_{\alpha}+b_{\alpha}\right) .
$$

This is clearly impossible when $s \gg 0$.

8.2. Projectives and injectives in $\mathcal{O}_{q}$. The results in Section 8.1 all have direct analogues in $\mathcal{O}_{q}$. As the proofs for $\mathcal{O}_{q}$ go exactly as for $\overline{\mathcal{O}}_{p}$ we only give the statements.

Theorem 8.4. For each $\lambda \in X^{-}$there exists a projective indecomposable module $P_{q}(\lambda) \in \mathcal{O}_{q}$ with head $L_{q}(\lambda)$. Moreover, $P_{q}(\lambda)$ is self-dual and is therefore also the injective envelope of $L_{q}(\lambda)$ and coincides with the $\infty$-tilting module $T_{q}^{\infty}\left(w_{0} \cdot \lambda\right)$.

Corollary 8.5. If $\lambda \in-\rho+X^{+}$then the socle of $\Delta_{q}(\lambda)$ equals $L_{q}\left(w_{0} \cdot \lambda\right)$.

Theorem 8.6. If $P_{q}$ is a projective module in $\mathcal{O}_{q}$ then $\operatorname{Hom}_{\mathcal{O}_{q}}\left(P_{q}, L_{q}(\lambda)\right)=0$ for all $\lambda \in$ $X \backslash X^{-}$. Likewise, when $\lambda \notin X^{-}$an injective module $I$ in $\mathcal{O}_{q}$ cannot contain $L_{q}(\lambda)$.

8.3. Reciprocity laws. The correspondence between the projective covers of simple modules with antidominant highest weights and the indecomposable $\infty$-tilting modules with highest weights in the closure of the dominant chamber gives rise to some reciprocity laws. To deduce these we need the following result (valid by similar arguments also in $\overline{\mathcal{O}}_{p}$ ).

Lemma 8.7. Let $\lambda, \mu \in X$. Then

(1) $\operatorname{Hom}_{\mathcal{O}_{q}}\left(\Delta_{q}(\lambda), \nabla_{q}(\mu)\right) \simeq\left\{\begin{array}{l}K \text { if } \lambda=\mu \\ 0 \text { otherwise }\end{array}\right.$ 
(2) $\operatorname{Ext}_{\mathcal{O}_{q}}^{1}\left(\Delta_{q}(\lambda), \nabla_{q}(\mu)\right)=0$.

Note that the Ext in (2) should be interpreted as Yoneda-Ext (we proved in Theorem 8.6 that $\mathcal{O}_{q}$ does not have enough projectives/injectives).

Proof. This goes by the standard arguments: The universal property of Verma modules (6.3) shows that if $\operatorname{Hom}_{\mathcal{O}_{q}}\left(\Delta_{q}(\lambda), \nabla_{q}(\mu)\right) \neq 0$ then $\mu \geq \lambda$. Dually, we get $\lambda \geq \mu$. Also the statement $\operatorname{Hom}_{\mathcal{O}_{q}}\left(\Delta_{q}(\lambda), \nabla_{q}(\lambda)\right)=K$ follows immediately from (6.3).

Now suppose we have an extension

$$
0 \rightarrow \nabla_{q}(\mu) \rightarrow E \rightarrow \Delta_{q}(\lambda) \rightarrow 0
$$

in $\mathcal{O}_{q}$. Unless $\mu>\lambda$ we get from (6.3) a homomorphism $\Delta_{q}(\lambda) \rightarrow E$, which splits (8.4). On the other hand, if $\mu>\lambda$ then the same argument splits the sequence dual to (8.4).

Recall from Section 4.1 the notion of a $\left(p^{r}, \Delta\right)$-filtration for a module $M$ in $\overline{\mathcal{O}}_{p}$ and the notation $\left(M: \bar{L}_{p}\left(\mu^{0}\right) \otimes \bar{\Delta}_{p}\left(\mu^{1}\right)^{(r)}\right)$ for the number of occurrences of $\bar{L}_{p}\left(\mu^{0}\right) \otimes \bar{\Delta}_{p}\left(\mu^{1}\right)^{(r)}$ in such a filtration. Note that if $\mu$ is antidominant and $r$ is large then $\bar{L}_{p}\left(\mu^{0}\right) \otimes \bar{\Delta}_{p}\left(\mu^{1}\right)^{(r)}=\bar{L}_{p}(\mu)$, see Corollary 6.2. Dually we have $\left(p^{r}, \nabla\right)$-filtrations with analogous notation. Of course we have $\left(M: \bar{L}_{p}\left(\mu^{0}\right) \otimes \bar{\Delta}_{p}\left(\mu^{1}\right)^{(r)}\right)=\left(D_{p} M: \bar{L}_{p}\left(\mu^{0}\right) \otimes \bar{\nabla}_{p}\left(\mu^{1}\right)^{(r)}\right)$.

We get the following reciprocity law in $\overline{\mathcal{O}}_{p}$.

Theorem 8.8. Let $\lambda, \mu \in X$ and suppose $\lambda+\rho \in X^{+}$. Then

$$
\left(\bar{T}_{p}^{\infty}(\lambda): \bar{\Delta}_{p}(\mu)\right)=\left(\bar{\Delta}_{p}(\mu): \bar{L}_{p}\left(w_{0} \cdot \lambda\right)\right) .
$$

Proof. Lemma 8.7 implies that

$$
\left(\bar{T}_{p}^{\infty}(\lambda): \bar{\Delta}_{p}(\mu)\right)=\operatorname{dim}_{K} \operatorname{Hom}_{\overline{\mathcal{O}}_{p}}\left(\bar{T}_{p}^{\infty}(\lambda), \bar{\nabla}_{p}(\mu)\right) .
$$

By Theorem 8.1 we may replace $\bar{T}_{p}^{\infty}(\lambda)$ by $\bar{P}_{p}\left(w_{0} \cdot \lambda\right)$. The projectivity of $\bar{P}_{p}\left(w_{0} \cdot \lambda\right)$ gives (when applying $\operatorname{Hom}_{\overline{\mathcal{O}}_{p}}\left(\bar{P}_{p}\left(w_{0} \cdot \lambda\right),-\right.$ ) to a $\left(p^{r}, \nabla\right)$-filtration of $\left.\bar{\nabla}_{p}(\mu)\right)$ the identity

$$
\operatorname{dim}_{K} \operatorname{Hom}_{\overline{\mathcal{O}}_{p}}\left(\bar{P}_{p}\left(w_{0} \cdot \lambda\right), \bar{\nabla}_{p}(\mu)\right)=\left(\bar{\nabla}_{p}(\mu): \bar{L}_{p}\left(w_{0} \cdot \lambda\right)\right) .
$$

Here we have chosen $r$ so large that $\lambda+\rho \in X_{p^{r}}$ (so that $\bar{L}_{p}\left(w_{0} \cdot \lambda\right)=\bar{L}_{p}\left(w_{0} \lambda+p^{r} \rho\right) \otimes \bar{L}_{p^{r}}(-\rho)^{(r)}$, cf. Corollary 6.2).

In $\mathcal{O}_{q}$ we use the $\left(\ell, p^{r}, \nabla\right)$-filtrations of dual Verma modules to obtain the following analogous result.

Theorem 8.9. Let $\lambda, \mu \in X$ and suppose $\lambda+\rho \in X^{+}$. Then

$$
\left(T_{q}^{\infty}(\lambda): \Delta_{q}(\mu)\right)=\left(\Delta_{q}(\mu): L_{q}\left(w_{0} \cdot \lambda\right)\right)
$$

where the right hand side denotes the multiplicity of $L_{q}\left(w_{0} \cdot \lambda\right)$ in an $\left(\ell, p^{r}, \nabla\right)$-filtration of $\nabla_{q}(\mu)$, with $r$ chosen so large that $w_{0} \cdot \lambda+\ell p^{r} \rho \in X_{\ell p^{r}}$.

These reciprocity laws imply the following equivalence.

Corollary 8.10. The set of indecomposable tilting characters $\left\{\operatorname{ch} \bar{T}_{p}^{\infty}(\lambda) \mid \lambda+\rho \in X^{+}\right\}$, respectively $\left\{\operatorname{ch} T_{q}^{\infty}(\lambda) \mid \lambda+\rho \in X^{+}\right\}$, determines the set of irreducible characters in $\overline{\mathcal{O}}_{p}$, respectively $\mathcal{O}_{q}$, and vice versa. 
Proof. Combining Theorem 8.8 combined with Corollary 4.3 we get

$$
\left(\bar{T}_{p}^{\infty}(\lambda): \bar{\Delta}_{p}(\mu)\right)=\left[\tilde{\Delta}_{r}(\mu): \tilde{L}_{p^{r}}\left(w_{0} \cdot \lambda\right)\right] .
$$

for all $\lambda+\rho \in X^{+}$and $\mu \in X$. Because of the periodici ty

$$
\left.\left[\tilde{\Delta}_{r}(\nu): \tilde{L}_{p^{r}}(\eta)\right)\right]=\left[\tilde{\Delta}_{r}\left(\nu+p^{r} \rho\right): \tilde{L}_{p^{r}}\left(\eta+p^{r} \rho\right)\right],
$$

valid for all $\nu, \eta \in X$ we can always determine the multiplicity of a given simple $\bar{u}_{r} \bar{B}_{K}$-module in a baby Verma module by passing to a case where the simple module has antidominant highest weight. Hence equation (8.5) means that the characters $\left\{\operatorname{ch} \bar{T}_{p}^{\infty}(\lambda) \mid \lambda \in X^{-}\right\}$determines the composition factors of all baby Verma modules. This is equivalent to the determination of all simple $\bar{u}_{r} \bar{B}_{K}$-modules. In turn these determine all irreducible characters in $\overline{\mathcal{O}}_{p}$, see Corollary 3.12 The reverse implication works as well.

The proof in the quantum case is analogous.

Remark 8.11. If $p \geq 2 h-2$ (or whenever Donkin's conjecture holds) we can in Corollary 8.10 replace the $\infty$-tilting modules with the finite dimensional tilting modules with highest weights in $\left(p^{r}-1\right) \rho+X^{+}$, respectively $\left.\left(\ell p^{r}-1\right) \rho\right)+X^{+}$, see Theorems 7.7(2) and 7.9(2). This result should be compared to [31] where it is proved (without any condition on $p$ ) that there exists $r>1$ such that the finite dimensional indecomposable tilting modules with highest weights in $\left(p^{r}-1\right) \rho+X_{p}$ determine all (finite dimensional) simple modules.

\section{REFERENCES}

[1] P. Achar, S. Makisumi, S. Riche, and G. Williamson, Koszul duality for Kac-Moody groups and characters of tilting modules, J. Amer. Math. Soc. 32 (2019), 261-310.

[2] H. H. Andersen, Tensor products of quantized tilting modules, Comm. Math. Phys. 149(1992), 149 - 159.

[3] H. H. Andersen, Quantum Groups at roots of \pm 1 , Comm. in Algebra 24 (1996), 3269 - 3282.

[4] H. H. Andersen, A sum formula for algebraic groups, (Proc. of conference in honor of D. Buchsbaum, Roma 1998). J. Pure and Appl. Alg. 152 (2000), 17 - 40.

[5] H. H. Andersen, p-filtrations and the Steinberg module, J. Algebra 244 (2001), 664-683.

[6] H. H. Andersen, The strong linkage principle for quantum groups at roots of 1, J. Alg., Vol. 260 (2003), $2-15$.

[7] H.H. Andersen and V. Mazorchuk, Category O for quantum groups, European Mathematical Society. Journal. 17, 2 (2015) $405-431$.

[8] H.H. Andersen, P. Polo and K. Wen, Representations of quantum algebras, Invent. Math. 104 (1991), 1 59.

[9] H.H. Andersen and K. Wen, Representations of quantum algebras. The mixed case, J. Reine Angew. Math. 427 (1992), 35 - 50.

[10] I. Bernstein, I. Gelfand, S. Gelfand; A certain category of g-modules. Funkcional. Anal. i Prilozen. 10 (1976), no. 2, 1-8.

[11] C. P. Bendel, D. K. Nakano, C. Pillen, P. Sobaje, Counterexamples to the Tilting and (p, r)-Filtration Conjectures, J. Reine Angew. Math, 767, (2020), 193-202.

[12] C.P. Bendel, D.K. Nakano, C. Pillen, and P. Sobaje, On Donkin's Tilting Module Conjecture I: Lowering the Prime, arXiv:2103.14164.

[13] C. Curtis, Representations of Lie algebras of classical types with applications to linear groups, J. Math. Mech. 9 (1960), 307-326.

[14] S. Donkin, On tilting modules for algebraic groups, Math. Z. 212 (1993), 39-60.

[15] S. Doty, The strong linkage principle, Amer. J. Math. 111 (1989), 135-141. 
[16] P. Etingof, D. Kazhdan, Quantization of Lie bialgebras. VI. Quantization of generalized Kac-Moody algebras. Transform. Groups 13 (2008), no.3-4, 527-539.

[17] P. Fiebig, Periodicity of irreducible modular and quantum characters, arXiv:2102.09865.

[18] M. Finkelberg, Fusion categories. Ph.D. Thesis, Harvard University. (1993), 50 pp.

[19] J. Franklin, Homomorphisms between Verma Modules in Characteristic p, J. Alg. 112 (1988), 58 - 88.

[20] A. Frisk, V. Mazorchuk, Regular strongly typical blocks of $\mathcal{O}_{q}$. Comm. Math. Phys. 291 (2009), no. 2, $533-542$.

[21] J.E. Humphreys, Representations of semisimple Lie algebras in the BGG category O. Graduate Studies in Math. 94. American Mathematical Society, Providence, RI, 2008. 317 (1980), 157-199.

[22] J. C. Jantzen, Lectures on quantum groups, Graduate Studies in Mathematics, Volume 6 (1996), 266 pp.

[23] J.C. Jantzen, Representations of Algebraic Groups, Mathematical Surveys and Monographs 107, Second edition, American Mathematical Society (2003).

[24] M. Kashiwara and T. Tanisaki, Kazhdan-Lusztig conjecture for affine Lie algebras with negative level, Duke Math. J. 77 (1995), 21-62.

[25] M. Kashiwara and T. Tanisaki, Kazhdan-Lusztig conjecture for affine Lie algebras with negative level, II. Non-integral case, Duke Math. J. 84 (1996), 771-813.

[26] D. Kazhdan and G. Lusztig, Representations of Coxeter groups and Hecke algebras, Invent. math. 53 ((1979), 165-184.

[27] G. Lusztig, Modular representations and quantum groups. Classical groups and related topics (Beijing, 1987), Contemp. Math., 82 (1989), 59-77.

[28] G. Lusztig, Quantum groups at roots of 1, Geom. Ded. 35 (1990), 89-114.

[29] S. Riche and G. Williamson, S. Riche and G. Williamson, A simple character formula, arXiv:1904.08085

[30] S. Ryom-Hansen, A q-analogue of Kempf's vanishing theorem. Moscow Mathematical Journal 3 (2003), pages $173-187$.

[31] P. Sobaje, On character formulas for simple and tilting modules, Adv. in Math., 369, (2020), 107172,8 pp.

[32] R. Steinberg, Representations of algebraic groups, Nagoya Math. J. 22 (1963), 33-56.

Centre for Quantum Geometry (QM), Imada, SDU, Denmark

Email address: h.haahr.andersen@gmail.com 